\title{
Defects in leaf carbohydrate metabolism compromise acclimation to high light and lead to a high chlorophyll fluorescence phenotype in Arabidopsis thaliana
}

Jessica Schmitz ${ }^{1}$, Mark Aurel Schöttler ${ }^{2}$, Stephan Krueger ${ }^{1}$, Stefan Geimer ${ }^{3}$, Anja Schneider ${ }^{4}$, Tatjana Kleine ${ }^{4}$, Dario Leister ${ }^{4}$, Kirsten Bell ${ }^{1}$, Ulf-Ingo Flügge ${ }^{1}$ and Rainer E Häusler ${ }^{1^{*}}$

\begin{abstract}
Background: We have studied the impact of carbohydrate-starvation on the acclimation response to high light using Arabidopsis thaliana double mutants strongly impaired in the day- and night path of photoassimilate export from the chloroplast. A complete knock-out mutant of the triose phosphate/phosphate translocator (TPT; tpt-2 mutant) was crossed to mutants defective in (i) starch biosynthesis (adg1-1, pgm1 and pgi1-1; knock-outs of ADP-glucose pyrophosphorylase, plastidial phosphoglucomutase and phosphoglucose isomerase) or (ii) starch mobilization (sex1-3, knock-out of glucan water dikinase) as well as in (iii) maltose export from the chloroplast (mex1-2).

Results: All double mutants were viable and indistinguishable from the wild type when grown under low light conditions, but - except for sex1-3/tpt-2 - developed a high chlorophyll fluorescence (HCF) phenotype and growth retardation when grown in high light. Immunoblots of thylakoid proteins, Blue-Native gel electrophoresis and chlorophyll fluorescence emission analyses at 77 Kelvin with the adg1-1/tpt-2 double mutant revealed that HCF was linked to a specific decrease in plastome-encoded core proteins of both photosystems (with the exception of the PSII component cytochrome $b_{559}$ ), whereas nuclear-encoded antennae (LHCS) accumulated normally, but were predominantly not attached to their photosystems. Uncoupled antennae are the major cause for HCF of darkadapted plants. Feeding of sucrose or glucose to high light-grown adg1-1/tpt-2 plants rescued the HCF- and growth phenotypes. Elevated sugar levels induce the expression of the glucose-6-phosphate/phosphate translocator2 (GPT2), which in principle could compensate for the deficiency in the TPT. A triple mutant with an additional defect in GPT2 (adg1-1/tpt-2/gpt2-1) exhibited an identical rescue of the HCF- and growth phenotype in response to sugar feeding as the adg1-1/tpt-2 double mutant, indicating that this rescue is independent from the sugar-triggered induction of GPT2.
\end{abstract}

Conclusions: We propose that cytosolic carbohydrate availability modulates acclimation to high light in $A$. thaliana. It is conceivable that the strong relationship between the chloroplast and nucleus with respect to a co-ordinated expression of photosynthesis genes is modified in carbohydrate-starved plants. Hence carbohydrates may be considered as a novel component involved in chloroplast-to-nucleus retrograde signaling, an aspect that will be addressed in future studies.

\footnotetext{
* Correspondence: rainer.haeusler@uni-koeln.de

${ }^{1}$ University of Cologne, Botanical Institute, Biocenter Cologne, Zülpicher Str.

47B, D-50674 Cologne, Germany

Full list of author information is available at the end of the article
} 


\section{Background}

The majority of the $\mathrm{CO}_{2}$ assimilated in the CalvinBenson cycle is eventually converted into carbohydrates. These can be retained inside the chloroplast in form of transitory starch or exported from the chloroplast and further transported via the phloem in form of sucrose to supply sink tissues such as roots, flowers and developing seeds with carbon and energy. The triose phosphate/ phosphate translocator (TPT) of the inner envelope membrane of chloroplasts is the major interface for the day path of photoassimilate export from the stroma [1]. Triose phosphates exported in the light are used as precursors for sucrose biosynthesis in the cytosol. In the night path of photoassimilate export, transitory starch is degraded via $\beta$-amylase, isoamylase and disproportionating enzyme (DPE1) yielding maltose and glucose as end products $[2,3]$. The transporters responsible for the export of glucose and maltose have been identified and the respective mutants characterized [4-7]. In the cytosol, maltose is further metabolized via DPE2 [8,9], a cytosolic heteroglycan [10] and cytosolic glucan phosphorylase (in A. thaliana PSH2) resulting in glucose and glucose-1phosphate (Glc1P), which enter further metabolism [11].

Surprisingly, an $A$. thaliana knock-down TPT mutant (tpt-1; identical to ape2, [acclimation of photosynthesis to environment2; [12]) lacked any pronounced development or growth phenotype [13], mainly because the reduced TPT transport capacity can be compensated for by an increased starch turnover in the light $[13,14]$ like previously reported for antisense TPT tobacco plants [15]. Hence, sucrose biosynthesis can be maintained in the light by the night path of photoassimilate export, i.e. starting from maltose and glucose. Strikingly, crosses of tpt-1 with adg1-1 [16] or sex1-1 [17], defective in the catalytic subunit of ADPglucose phosphorylase (AGPase) or glucan water dikinase (GWD, [18]), were still viable albeit retarded in growth, suggesting that the residual TPT activity permits survival of the plants [13]. In particular, the adg1-1/tpt-1 double mutant exhibited a severe growth and photosynthesis phenotype reminiscent of potato plants with an antisense inhibition of both the TPT and AGPase [19]. The A. thaliana double mutant is characterized by impaired photosynthetic electron transport, changes in the chloroplast ultrastructure, such as grana hyperstacking, and increased numbers of plastoglobules, high Chl fluorescence (HCF) in the dark-adapted state, and a perturbed redox equilibrium in the light and dark [20]. It has been proposed that residual TPT activity in the tpt-1 allele permits survival of the adg1-1/tpt-1 double mutant [20].

A full knock-out mutant of the TPT gene (i.e. tpt-2) has been isolated and crossed not only to adg1-1, but also to further mutants defective in starch biosynthesis, its mobilization or the export of the starch degradation product maltose from the chloroplasts. All double mutants were viable and exhibited a pronounced growth and/or photosynthesis phenotype only when grown under high light, but not under low light conditions.

Here we have focused on the mechanistic basis of the HCF phenotype and the role of soluble sugars in the acclimation to high light intensities in mutants compromised in carbohydrate metabolism. The HCF phenotype of the adg1-1/tpt-1 double mutant allele could only be partially explained by an increased rate of chlororespiration and the concomitant enhanced reduction state of the plastoquinone pool in the dark. This notion was mainly based on the additional increase in the ground Chl- $a$ fluorescence $\left(\mathrm{F}_{\mathrm{o}}\right)$ in the absence of $\mathrm{O}_{2}$ or following the application of octyl gallate, an inhibitor of the plastid localized alternative oxidase, a central component involved in chlororespiration [20]. The mechanistic basis for the HCF phenotype has now been resolved. It is mainly caused by light harvesting complexes (LHCs) uncoupled from their reaction centers.

The roles of soluble sugars and the glucose-6-phosphate/phosphate translocator2 (GPT2) in the altered acclimation response of $a d g 1-1 / t p t-2$ to high light has been addressed by feeding sucrose or glucose to the double mutant or an adg1-1/tpt-2/gpt2-1 triple mutant. GPT2 has been shown to be strongly induced both at a transcriptional and functional level in leaves of mutants incapable of sufficient starch biosynthesis, such as adg11, pgm1 or pgi1-2 as well as in adg1-1/tpt-1 [21]. The induction of GPT2 correlates with the accumulation of soluble sugars in the mutant's background. Our data demonstrate that soluble sugars are key players in the compromised or modified acclimation response to high light in $a d g 1-1 / t p t-2$ and that GPT2 is not involved in this response.

\section{Results}

Isolation of a knock-out mutant of the TPT gene (tpt-2) and generation of double mutants

The tpt-1 mutant (WS-2 background), carrying a T-DNA insertion in the promoter region $24 \mathrm{bp}$ upstream of the start ATG, showed residual transcripts of the TPT gene and substantial TPT transport activities of up to $16 \%$ of the wild type $[13,21]$. In order to study the consequences of a complete knock-out of the TPT gene in A. thaliana, we isolated the tpt-2 allele (Col-0 background; N573707; SALK_073707.54.25.x), which carries a T-DNA insertion 8 bp downstream of the start ATG (Figure 1A). The tpt-2 mutant plants completely lacked TPT specific transcripts (Figure $1 \mathrm{~B}$ ), and $\mathrm{P}_{\mathrm{i}}$ transport decreased by $44 \%$ from $2.94 \pm 0.35 \mathrm{nmol} \cdot \mathrm{g}^{-1} \mathrm{fw}$ in the wild type to $1.64 \pm$ $0.02 \mathrm{nmol} \cdot \mathrm{g}^{-1} \mathrm{fw}$ in tpt-2. Transport of 3-PGA (3.07 \pm $0.41 \mathrm{nmol} \cdot \mathrm{g}^{-1} \mathrm{fw}$ in wild-type plants) was not detectable in tpt-2, demonstrating that the tpt-2 allele represents a 


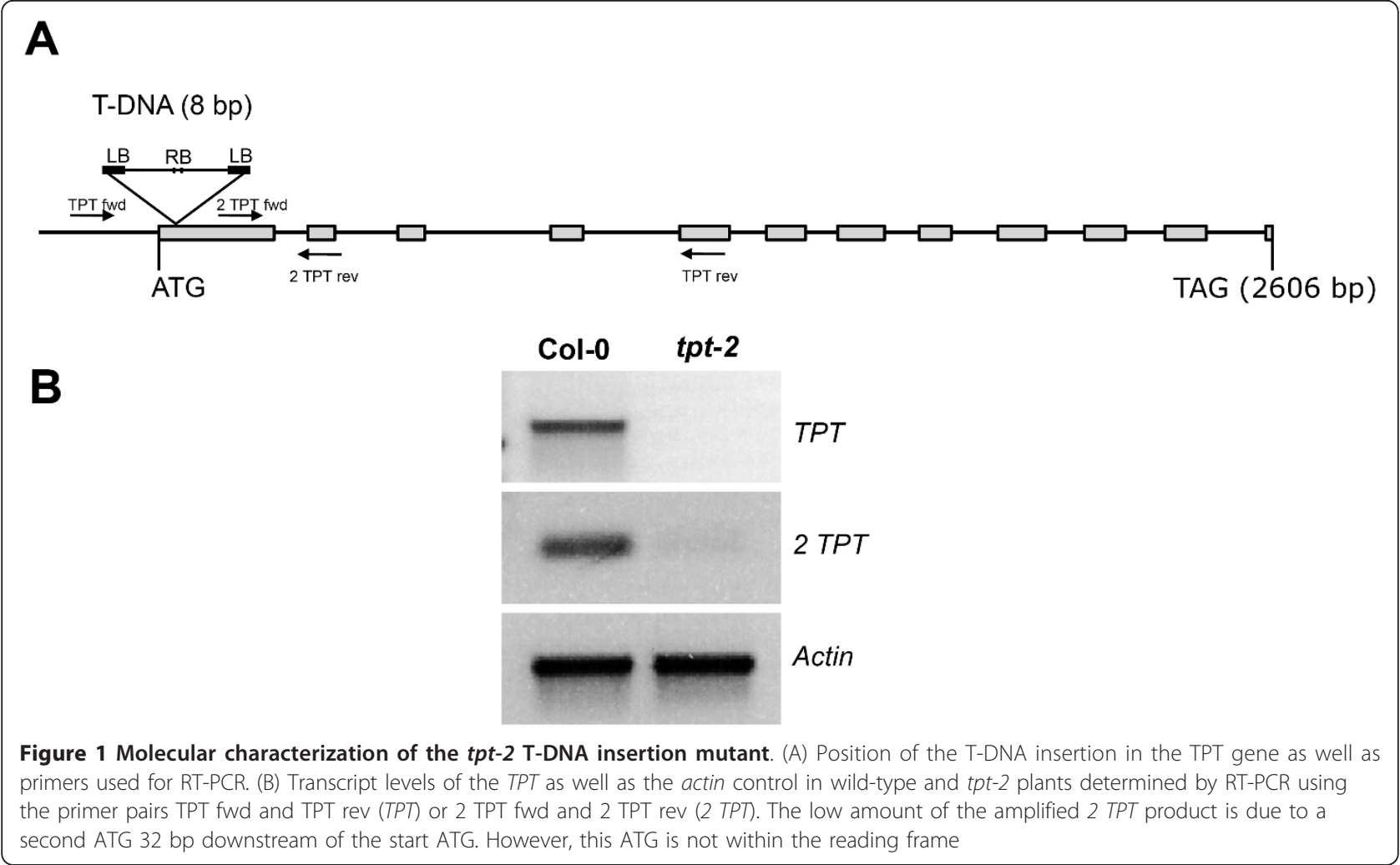

loss-of-function mutant of the TPT (the above data express the mean $\pm \mathrm{SE}$ of $\mathrm{n}=4$ experiments). In order to simultaneously block the day- and night path of carbon export from the chloroplasts, the tpt-2 mutant has been crossed to the starch-free or low-starch mutants adg1-1 [16], pgm1 [22] and pgi1-1 [23] as well as to mutants compromised in starch mobilization (sex1-3, [24]) or export of maltose from the chloroplast (mex 1-2, [5]). Despite the complete loss of TPT activity all double mutants are viable.

The phenotypes of adg1-1/tpt-2 emerged only under high light conditions

Like adg1-1/tpt-1 the adg1-1/tpt-2 double mutant allele is retarded in vegetative growth and shows a HCF phenotype in the dark-adapted state [20]. Both phenotypes emerged when double mutant plants were grown at a photosynthetic photon flux density (PDF) well above 50-70 $\mu \mathrm{mol} \cdot \mathrm{m}^{-2} \cdot \mathrm{s}^{-1}$. However, at a PFD below $50 \mu \mathrm{mol} \cdot \mathrm{m}^{-2} \cdot \mathrm{s}^{-1}$, adg1-1/tpt-2 lacked any prominent phenotype. An impaired day and night path of carbon export from the chloroplast compromises the redox equilibrium in the stroma and photosynthesis. Both are strongly dependent on the light intensity the plants experience during growth. Here we compared the characteristics of mutant and wild-type plants grown under low light (LL) conditions (i.e. a PFD of $30 \mu \mathrm{mol} \cdot \mathrm{m}^{-2} \cdot \mathrm{s}^{-1}$ ) with those grown at a ten-fold higher PFD (i.e. $300 \mu \mathrm{mol} \cdot \mathrm{m}^{-2} \cdot \mathrm{s}^{-1}$ ), defined as high light (HL) conditions. All plant lines were considerably smaller when grown in LL (Figure 2B) compared to HL (Figure 2A) and adg11/tpt-2 grown in LL lacked any pronounced growth- or HCF phenotype (Figure 2B and 2D; Table 1A). However, the characteristic phenotypes of the double mutant were well marked in HL-grown plants (Figure 2A and 2C). Vegetative growth was substantially diminished (Figure 2A) and dark-adapted adg1-1/tpt-2 plants exhibited a characteristic increase in the ground fluorescence $\left(F_{o}\right)$, which was reflected in a decrease in the $F_{v} / F_{m}$ ratio from 0.8 in wild-type or single mutant to around 0.3 in double mutant plants (Figure 2C, Table 1B). In contrast, LL-grown adg1-1/tpt-2 plants failed to exhibit a HCF phenotype during their development. In parallel with growth retardation and the HCF phenotype Chl-, carotenoid- and protein contents were diminished only in HLgrown double mutant compared to wild-type and single mutant plants (Table $1 \mathrm{~B}$ ). The $\mathrm{Chl} a / b$ ratio was decreased from around 3 in the wild type and the single mutants to 2.1 in HL-grown double mutants and was also slightly lower in LL-grown adg1-1/tpt-2 plants (Table 1A).

The growth and photosynthesis phenotypes of adg1-1/ tpt-2 were accompanied by an altered leaf anatomy and chloroplast ultrastructure compared to the wild type 


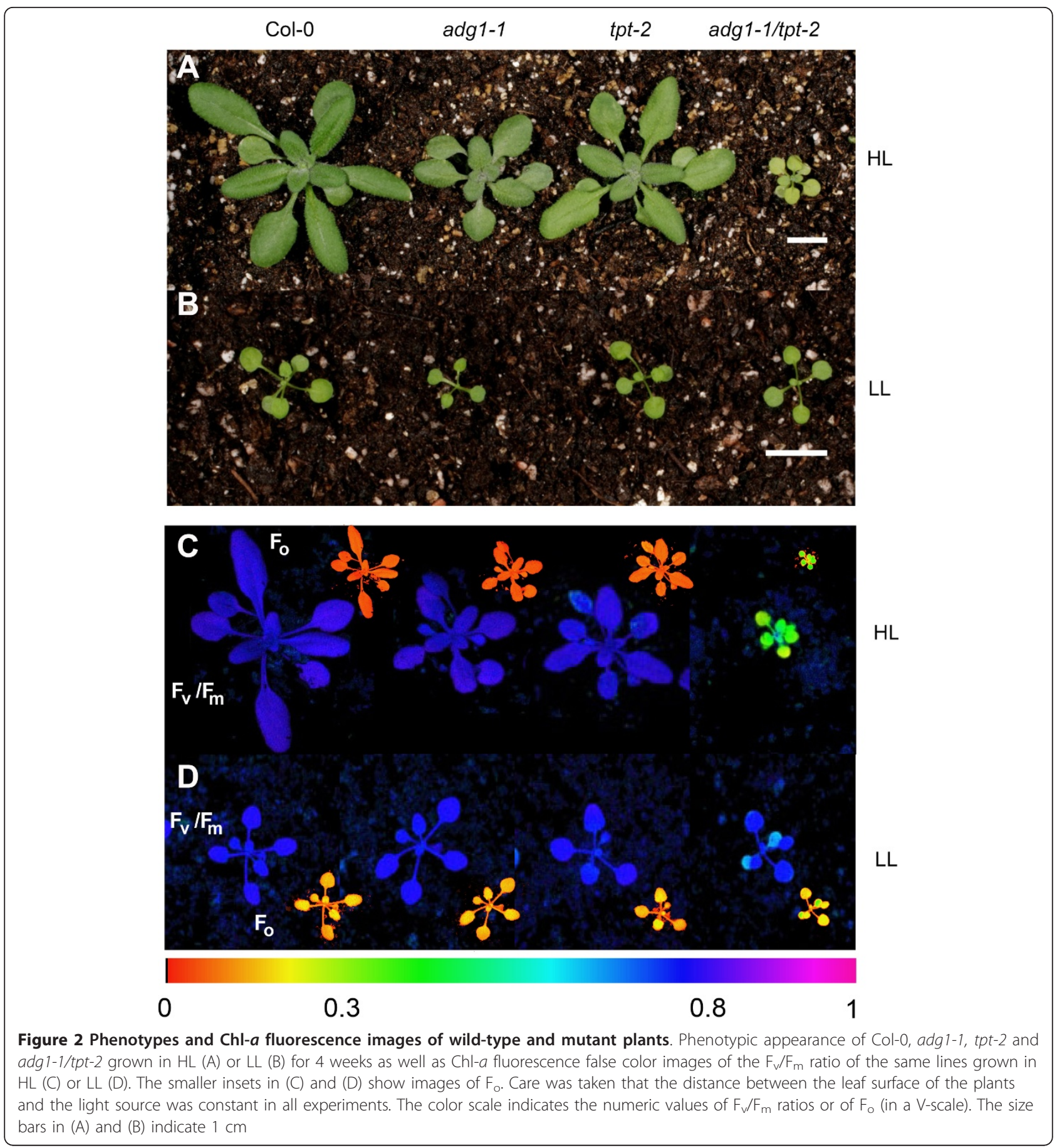

(Figure 3). In general, leaves of HL-grown plants were thicker (Figure $3 \mathrm{~B}$ and $3 \mathrm{~F}$ ) compared to those grown in LL (Figure 3A and 3E). However, compared to the wild type, HL-grown adg1-1/tpt-2 contained less, but larger mesophyll cells (Figure 3B and 3F).

With regard to the chloroplast ultrastructure, the wild type exhibited a higher number of grana stacks when grown under LL-conditions (Figure 3C, on average 5.1 stacks per granum) compared to HL-conditions (Figure 3D; on average 3.9 stacks per granum). Strikingly, grana stacking in chloroplasts of adg1-1/tpt-2 was even increased from 6.7 to 8.3 stacks per granum in LLcompared to HL-grown plants (Figure $3 \mathrm{G}$ to $3 \mathrm{~J}$ ). Additional File 1 shows a comparison of the distribution of 'grana stack number classes' between LL- and HLgrown wild-type and double mutant plants. Under both 
Table $1 \mathrm{Chl}-a$ fluorescence parameters as well as pigment and protein contents of the adg1-1/tpt-2 double mutant compared to the wild type (Col-0) and the adg1-1 and tpt-1 single mutants

\begin{tabular}{|c|c|c|c|c|c|c|}
\hline Lines & $\mathrm{F}_{\mathrm{v}} / \mathrm{F}_{\mathrm{m}}$ ratio & ФPSII & Chl content $\left(\mathrm{mg} \cdot \mathrm{m}^{-2}\right)$ & $\mathrm{Chl} a / b$ ratio & Carotenoid content $\left(\mathrm{mg} \cdot \mathrm{m}^{-2}\right)$ & Protein content $\left(\mathrm{g} \cdot \mathrm{m}^{-2}\right)$ \\
\hline \multicolumn{7}{|c|}{ A Plants grown in LL } \\
\hline Col-0 & $0.744 \pm 0.011$ & $0.584 \pm 0.016^{\mathbf{b}, \mathbf{d}}$ & $127.2 \pm 7.5$ & $2.68 \pm 0.03^{d}$ & $4.4 \pm 0.3$ & $0.59 \pm 0.10$ \\
\hline $\operatorname{adg} 1-1$ & $0.751 \pm 0.010$ & $0.487 \pm 0.077^{\mathrm{a}, \mathrm{c}}$ & $133.8 \pm 16.6$ & $2.64 \pm 0.06^{d}$ & $4.5 \pm 0.6$ & $0.57 \pm 0.14$ \\
\hline tpt-2 & $0.753 \pm 0.019$ & $0.582 \pm 0.030^{\mathbf{b}, \mathbf{d}}$ & $135.8 \pm 11.3$ & $2.61 \pm 0.08^{d}$ & $4.5 \pm 0.3$ & $0.44 \pm 0.04$ \\
\hline$a d g 1-1 / t p t-2$ & $0.746 \pm 0.005$ & $0.472 \pm 0.022^{a, c}$ & $135.3 \pm 9.2$ & $2.35 \pm 0.07^{\mathbf{a}, \mathbf{b}, \mathbf{c}}$ & $4.7 \pm 0.4$ & $0.54 \pm 0.05$ \\
\hline \multicolumn{7}{|c|}{ B Plants grown in $\mathrm{HL}$} \\
\hline Col-0 & $0.800 \pm 0.007^{c, d}$ & $0.396 \pm 0.014^{\mathbf{d}}$ & $286.2 \pm 26.5^{b, c, d}$ & $3.09 \pm 0.13^{d}$ & $10.6 \pm 0.5^{\mathbf{b}, \mathbf{d}}$ & $3.38 \pm 0.42^{\mathbf{b}, \mathbf{d}}$ \\
\hline $\operatorname{adg1-1}$ & $0.778 \pm 0.007^{\mathbf{d}}$ & $0.366 \pm 0.014^{\mathbf{d}}$ & $320.6 \pm 24.3^{a, c, d}$ & $3.00 \pm 0.04^{c, d}$ & $12.4 \pm 0.9^{a, c, d}$ & $4.29 \pm 0.64^{\text {a, c, d }}$ \\
\hline tpt-2 & $0.757 \pm 0.030^{\text {a, d }}$ & $0.359 \pm 0.046^{\mathbf{d}}$ & $258.7 \pm 9.9^{\mathbf{a}, \mathbf{b}, \mathbf{d}}$ & $3.11 \pm 0.07^{\mathbf{b}, \mathbf{d}}$ & $10.1 \pm 0.6^{\mathbf{b}, \mathbf{d}}$ & $3.24 \pm 0.42^{\mathbf{b}, \mathbf{d}}$ \\
\hline adg1-1/tpt-2 & $0.226 \pm 0.021^{\text {a, b, }} \mathbf{c}$ & $0.056 \pm 0.027^{\text {a, b, c }}$ & $103.0 \pm 10.8^{\text {a, b, c }}$ & $2.13 \pm 0.04^{\text {a, b, c }}$ & $5.5 \pm 0.5^{\text {a, b, c }}$ & $2.26 \pm 0.48^{a, b, c}$ \\
\hline
\end{tabular}

The plants were grown for 4 weeks either in $\mathrm{LL}(\mathrm{A})$ or $\mathrm{HL}(\mathrm{B})$. Photosynthesis parameters were determined with the Imaging-PAM fluorometer using the integrated light source. Before the determination of $\mathrm{F}_{\mathrm{v}} / \mathrm{F}_{\mathrm{m}}$ ratios the plants were dark-adapted for $15 \mathrm{~min}$. The $\Phi P S I I$ values refer to steady state photosynthesis, which was attained 7-10 min after onset of illumination with PDFs similar to those the plants experienced during growth (i.e. at $30 \mu \mathrm{molm} \mathrm{m}^{-2} \cdot \mathrm{s}^{-1}$ [LL] and $300 \mu \mathrm{mol} \cdot \mathrm{m}^{-2} \cdot \mathrm{s}^{-1}[\mathrm{HL}]$, respectively). The data represent the mean $\pm S E$ of $n=3\left(F_{v} / F_{m}\right.$ ratio, $\left.\Phi P S I I\right)$ or $n=5$ (Chl content, Chl a/b ratio, carotenoid- and protein content) measurements. Significant differences in the data pairs were identified by the ANOVA test and further analyzed by the Tukey-Kramer test. The letters as superscripts denote the lines to which a significant difference $(P<0.05)$ was found with $\mathrm{a}=\mathrm{Col}-0, \mathrm{~b}=$ adg1-1, $\mathrm{c}=$ tpt-2 and $\mathrm{d}=$ adg1-1/tpt-2. 


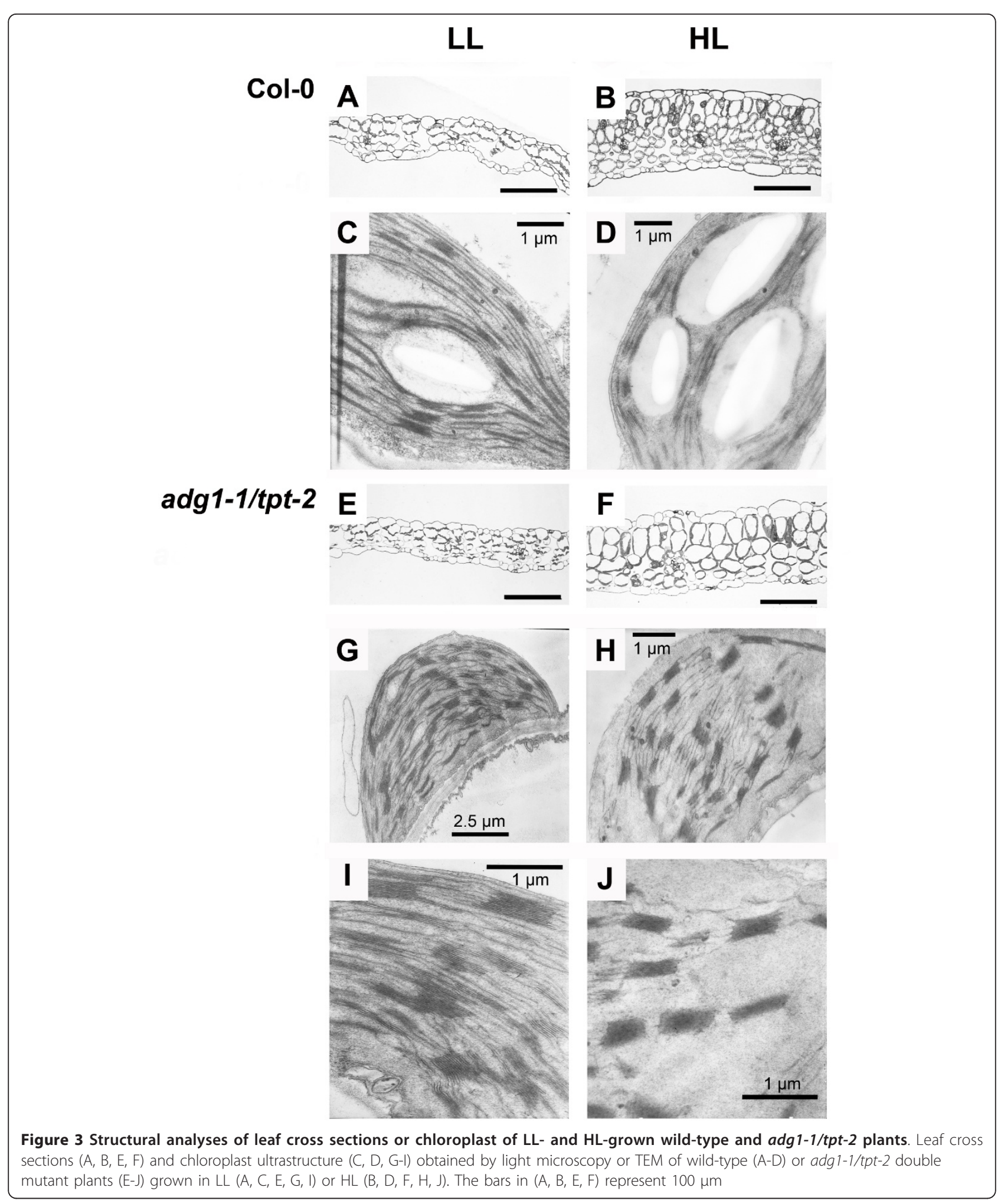


growth light conditions adg1-1/tpt-2 showed a much broader distribution of 'grana stack number classes' with up to 17 stacks per granum in HL-grown plants compared to the wild type with a maximum of 9 stacks per granum.

Taken together, the above data indicate that the phenotypic changes in adg1-1/tpt-2 are light dependent and suggest that mechanisms leading to a proper acclimation to $\mathrm{HL}$ are compromised in the double mutant compared to wild-type or single mutant plants.

\section{Composition and abundance of photosynthetic} multiprotein complexes and supercomplexes are strongly altered in HL-grown adg1-1/tpt-2 plants

Taking the HCF phenotype and increased grana stacking in both the adg1-1/tpt-1 and adg1-1/tpt-2 alleles into account, we investigated the accumulation of photosynthesis-related proteins and corresponding multiportein complexes by immunoblot analysis (Figure $4 \mathrm{~A}$ and $4 \mathrm{C}$ ) and by Blue-Native PAGE (Figure 4D). HL-grown adg1-1/tpt-2 plants exhibited substantial changes in the abundance of PSII core components compared to the wild type and the single mutants. The plastome-encoded subunits PsbB (CP47), PsbD (D2), PsbA (D1), and PsbC (CP43) were hardly detectable in the double mutant, whereas the abundance of PSII-associated Lhcb proteins, as well as the oxygen evolving complex (OEC; PsbO), which are nuclearencoded proteins, remained unaffected. In contrast, when plants were grown in LL, the PSII core protein contents in adg1-1/tpt-2, wild-type and single mutant plants were very similar (Figure 4A). The Cyt $\mathrm{b}_{6} / \mathrm{f}$ complex component PetC (Rieske protein) was decreased in the HL-grown

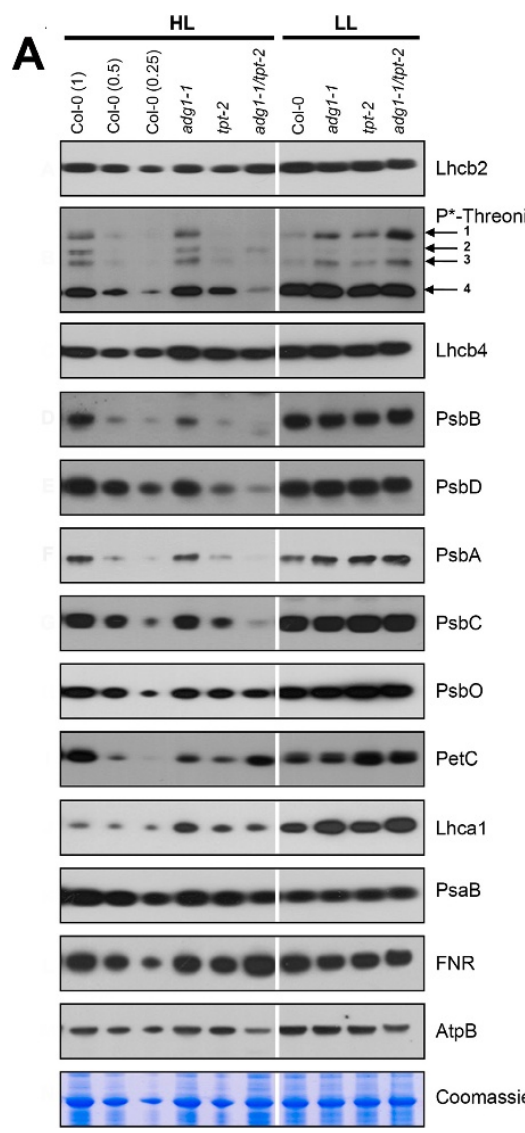

B

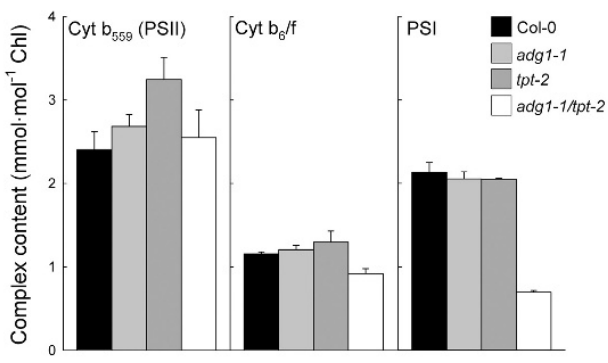

C

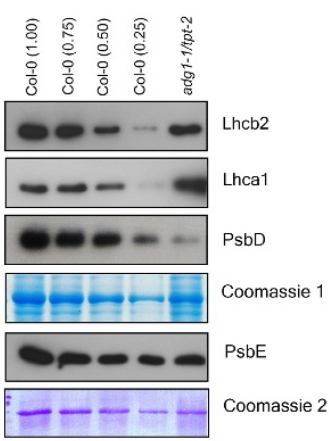

D

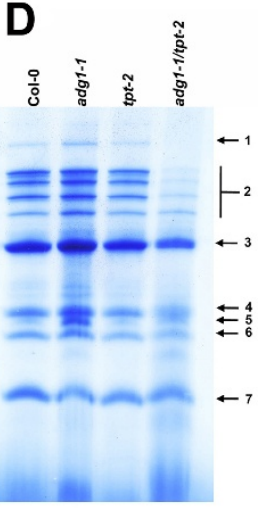

Figure 4 Immunoblots of thylakoid proteins, spectroscopic determinations of functional PS components, and separation of PS complexes by Blue-Native gel electrophoresis. (A) Immunoblots of thylakoid proteins associated with photosynthesis after separation of total proteins (approximately $10 \mathrm{\mu g}$ per lane) isolated from leaves of $\mathrm{HL}$ - and LL- grown Col-0 wild type, the adg 1-1 and tpt-2 single mutants as well as the adg 1-1/ tpt-2 double mutant on SDS-PAGE. Note that the Coomassie staining for proteins of HL-grown tpt-2 corresponds to about 0.5 -fold rather than 1 -fold of Col-0 protein. $P^{*}$-Threonin indicates signals obtained following incubation of the blots with a phospho-threonin antibody. The numbers indicate signals from PsbC (1), CaS (2), PsbA/PsbD (3), and Lhcbll (4). (B) Spectroscopic determinations of functional components of PSII, PSI and the cyt $b_{6} / f$ complex. (C) Immunoblots of PsbE in comparison to Lhcb2, Lhca1, and PsbE in HL-grown wild-type and adg1-1/tpt-2 plants. (D) Separation of thylakoid proteins of HL-grown Col-0 wild type, the adg 1-1 and tpt-2 single mutants as well as the adg 1-1/tpt-2 double mutant on Blue-Native gels. The numbers indicate individual protein fractions of the isolated thylakoids such as the NDH complex (1, see Additional file 2: Table S2), PSII super complexes (2), PSI supercomplex/PSII dimer (3), ATPase (4), PSII monomers (5), PSII/PsbC (6), and LHCII trimers (7) 
double mutant as well as in the single mutants compared to the wild type (Figure 4A). The plastome-encoded PSI core component PsaB (P700 apoprotein A2), which together with PsaA (P700 apoprotein A1) is involved in electron transfer from plastocyanin to the PSI acceptor side [25], was diminished by 50-70\% in HL-grown adg1-1/ tpt-2, but remained unchanged in the single mutants irrespectively of the growth PDF. Nuclear-encoded Lhca1 was increased in all HL-grown mutant lines compared to the wild type (Figure 4A). Moreover, ferredoxin:NADP reductase (FNR), which is also nuclear-encoded, was increased in the double mutant grown under HL-condition (Figure 4A), whereas, under HL- and LL-conditions, the abundance of the plastome-encoded ATPase subunit AtpB was slightly decreased in adg1-1/tpt-2.

Furthermore, the phosphorylation state of several thylakoid proteins was analyzed. Phosphorylation levels of Lhcb2, CP43, CaS (a calcium sensing receptor residing in the stroma thylakoids; [26]), and D1/D2 were substantially diminished in HL-grown adg1-1/tpt-2 compared to wild-type and single mutant plants (Figure 4A). The decrease in the D1/D2 phosphorylation state correlates well with its overall diminished protein content, whereas the phosphorylation state of Lhcb2 was decreased despite unaffected protein levels (Figure 4A).

Spectroscopic analyses of functional components of the photosynthetic electron transport chain in isolated thylakoids supported the data obtained by immunoblots (Figure 4B). PSI contents were diminished by more than $50 \%$ only in HL-grown double mutants, but not in the single mutants compared to the wild type. These data are well in line with immunoblots against the PSI core component PsaB (Figure 4A). The redox-active Cyt $\mathrm{b}_{6} / \mathrm{f}$ complex was decreased in HL-grown adg1-1/tpt-2 compared to wild-type and single mutant plants. Accumulation of $C y t b_{559}$, an essential structural component of PSII, was unchanged in HL-grown double mutant plants (Figure 4B) despite the overall decrease in PSII core components. Immunoblots of the PsbE protein, which forms the $\alpha$-subunit of $C y t b_{559}$, support the spectroscopic determination of Cyt $b_{559}$. The decrease in PsbE was much less pronounced compared for instance to PsbD in HL-grown adg1-1/tpt-2 (Figure 4C).

The impaired abundance of PSII core components and the formation of supercomplexes in adg1-1/tpt-2 was further supported by separation of thylakoid proteins isolated from HL-grown plants on Blue-Native gels. Most strikingly the abundance of PSII and PSI supercomplexes as well as of PSII monomers was severely diminished in adg1-1/tpt-2 compared to the wild type and the single mutants, whereas ATPase and LHCII trimers (which serve as a reference at $110 \mathrm{kDa}$ ) were not affected (Figure 4D). Moreover, the high molecular mass band 1 (>1000 kDa) was completely missing in the double mutant. The protein composition of band 1 was determined by LC-MS/MS (Additional File 2). It corresponds well with an earlier report by Peng et al. [27] and suggests that the NDH1-PSI supercomplex is absent in HL-grown adg1-1/tpt-2.

\section{Chl- $a$ fluorescence emission at 77 Kelvin reveals a high portion of free, highly fluorescent LHCs uncoupled from their photosystems in adg1-1/tpt-2}

The low temperature Chl- $a$ fluorescence emission from isolated thylakoids permits an estimate of the functional coupling of LHCs with the reaction centers. While the emission spectra of the wild type and the single mutants were almost identical (Figure $5 \mathrm{~A}$ to $5 \mathrm{C}$ ), there were characteristic changes in the spectrum obtained from adg1-1/tpt-2 (Figure 5D). The wild type and the single mutants show a maximum emission from PSII-LHCII at $686 \mathrm{~nm}$ with a shoulder at $692 \mathrm{~nm}$ emitted from CP47. These signals are typical for PSII-LHCII supercomplexes with highly efficient exciton transfer from the outer antennae to the reaction centers. However, in the double mutant the maximum emission is shifted from $686 \mathrm{~nm}$ to $684 \mathrm{~nm}$, reflecting a fraction of free LHCII excitonically uncoupled from PSII. Likewise, the emission maximum at $731 \mathrm{~nm}$ observed for the wild type and the single mutants is typical for a strong coupling of LHCI to PSI. In the double mutant the emission maximum is shifted from $731 \mathrm{~nm}$ to $722 \mathrm{~nm}$, which indicates a high portion of uncoupled LHCI with emission maxima between 705 and $725 \mathrm{~nm}$. These data indicate that a high portion of $\mathrm{F}_{\mathrm{o}}$ in the adg1-1/tpt-2 double mutant is emitted from LHCs uncoupled from their photosystems.

\section{The efficiency of PSI is less affected compared to PSII in HL-grown adg1-1/tpt-2 plants}

Previous determinations of photosynthesis parameters of the adg1-1/tpt-1 allele suggested a decrease in the electron transport rate (ETR) through PSII (ETRII) by nearly $90 \%$ compared to the wild type or the single mutants [13]. In view of the perturbed stoichiometry of both photosystems as well as a high portion of antennae uncoupled from their reaction centers we compared the quantum efficiencies of PSII and PSI electron transfer (i. e. ФPSII and (PPSI) rather than the derived ETRII and ETRI. The light curves of ФPSII and ФPSI were similar in LL- and HL-grown wild-type and single mutant plants (Figure $6 \mathrm{~A}$ to $6 \mathrm{C}$ and $6 \mathrm{E}$ to $6 \mathrm{G}$ ), but exhibited characteristic differences in the adg1-1/tpt-2 double mutant (Figure 6D and 6H). Up to a PFD of 200 $\mu \mathrm{mol} \cdot \mathrm{m}^{-2} \cdot \mathrm{s}^{-1}$ the ФPSII values were lower in $\mathrm{HL}$ - compared to LL-grown adg1-1/tpt-2 plants (Figure 6D). However, both curves converged at PFDs above 200 $\mu \mathrm{mol} \cdot \mathrm{m}^{-2} \cdot \mathrm{s}^{-1}$ and $\Phi$ PSII had similar values as LL-grown 


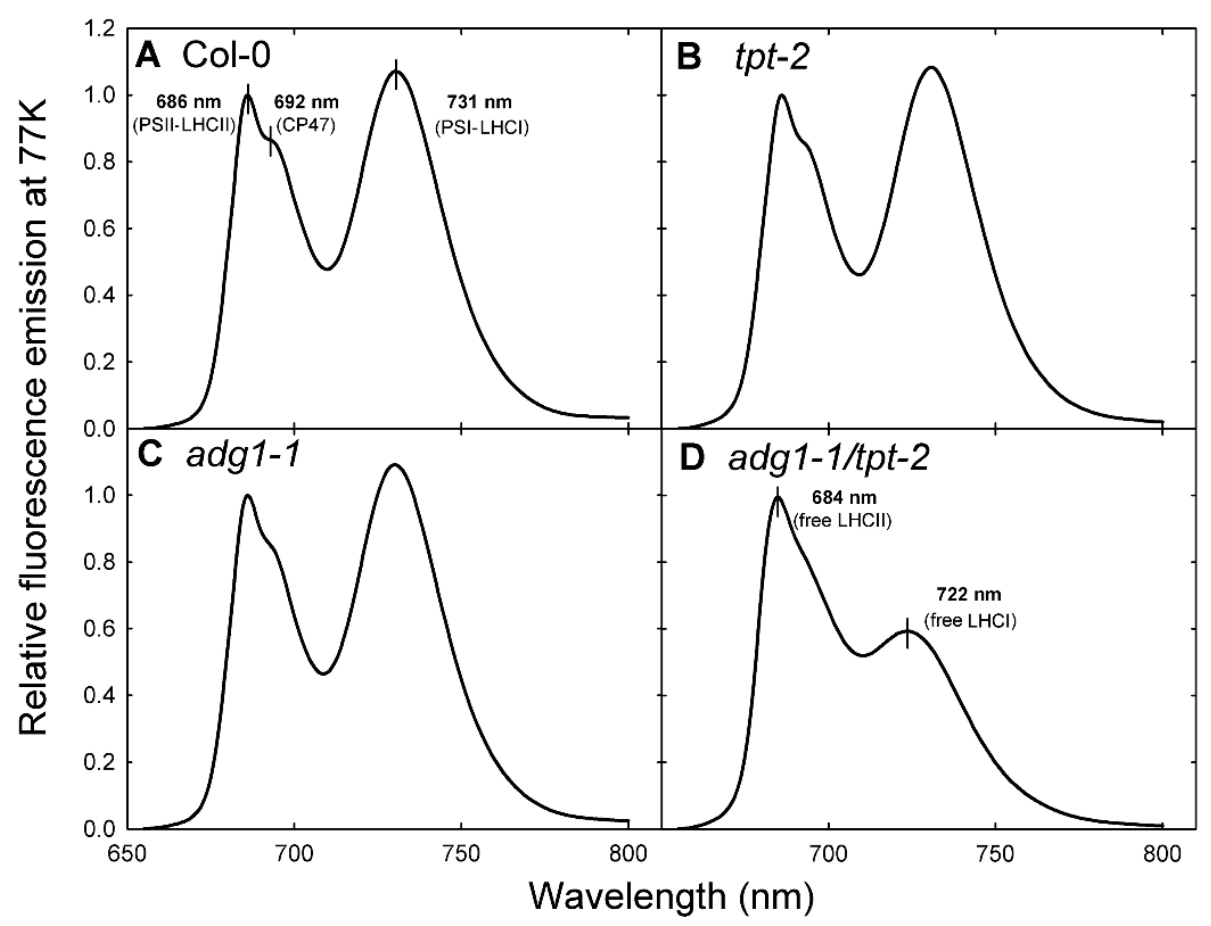

Figure 5 Fluorescence emission spectra of $\mathbf{C h l}$ at $\mathbf{7 7} \mathrm{K}$. Relative fluorescence emission spectra of $\mathrm{Chl}$ in isolated thylakoids from Col-0 wild type (A), tpt-2 (B), adg1-1 (C), and adg1-1/tpt-2 (D) determined at $77 \mathrm{~K}$. The spectra were normalized for the peak intensity at $686 \mathrm{~nm}$ in case of the wild type and the single mutants, and to the emission maximum of the free LHCII at $684 \mathrm{~nm}$ in case of the adg1-1/tpt-2 double mutant

wild-type and single mutant plants. In contrast to the ФPSII response to increasing PFDs, ФPSI values were appreciably higher in HL- compared to LL-grown double mutant plants throughout the whole range of PFDs applied (Figure 6H), suggesting that the efficiency of PSI was less affected compared to PSII in HL-grown double mutant plants.

Moreover, donor site limitation of PSI ( $\Phi$ ND, i.e. the limitation of electron transfer from the $\mathrm{cytb}_{6} / \mathrm{f}$ complex to PSI) was increased in HL-grown adg1-1/tpt-2 plants, compared to the wild type and the single mutants, while acceptor site limitation (ФNA, i.e. the limitation of electron transfer from PSI to NADP) was close to zero (Additional File 3). Both parameters remained unaffected between the lines, when the plants were grown in LL. Hence, in HL-grown double mutant plants electron transfer from PSII to PSI is compromised and PSI efficiency is not acceptor site limited.

\section{Far red and actinic illumination induces non-}

\section{photochemical quenching of HCF in adg 1-1/tpt-2}

The portion of the LHCs not associated with PSII or PSI core proteins gives rise to the increased Chl- $a$ fluorescence yield in dark-adapted HL-grown adg1-1/tpt-2. To separate individual components of the increased $F_{o}$ in adg1-1/tpt-2 the time-course routine outlined in Figure 7A was applied. Upon determination of $F_{o}$ and $F_{m}$, plants were illuminated with far red (FR) light to specifically excite PSI (in order to completely oxidize the PSII acceptor site) and subsequently with actinic light (AL). The adg1-1/tpt-2 double mutant exhibited a slow decline in the fluorescence yield in the presence of FR until a new minimum level was attained (Figure 7B), whereas $F_{o}$ in the wild type remained unaffected by $F R$ (Figure 7A). It is conceivable that this portion of quenching observed in the double mutant is due to reoxidation of $\mathrm{Q}_{\mathrm{A}}$. Upon turning FR off the fluorescence signal of adg1-1/tpt-2 relaxed again slowly to the original $F_{o}$ value observed in dark-adapted plants (Figure 7B). Following the application of strong AL (PFD of approximately $\left.4000 \mu \mathrm{mol} \cdot \mathrm{m}^{-2} \cdot \mathrm{s}^{-1}\right)$, the fluorescence signal of adg1-1/tpt-2 dropped rapidly below $\mathrm{F}_{\mathrm{o}}$ and reached a minimum $\left(\mathrm{F}_{\mathrm{o}}{ }^{\prime}\right)$ upon switching off AL (Figure $7 \mathrm{~B}$ ), which was similar to the $\mathrm{F}_{\mathrm{o}}$ observed in wild-type plants (Figure 7A), suggesting that this residual fluorescence was based on LHCs associated with the remaining PSII core components and that the fluorescence of LHCs not associated with PSII was quenched nonphotochemically. In contrast to the wild-type, $\mathrm{F}_{\mathrm{o}}$ in adg1-1/tpt-2 increased again upon darkening and the subsequent application of a saturating light pulse (SP) caused only a small increase in the fluorescence yield, supporting the idea that non-photochemical quenching (NPQ) of free LCHs is the major cause for the 


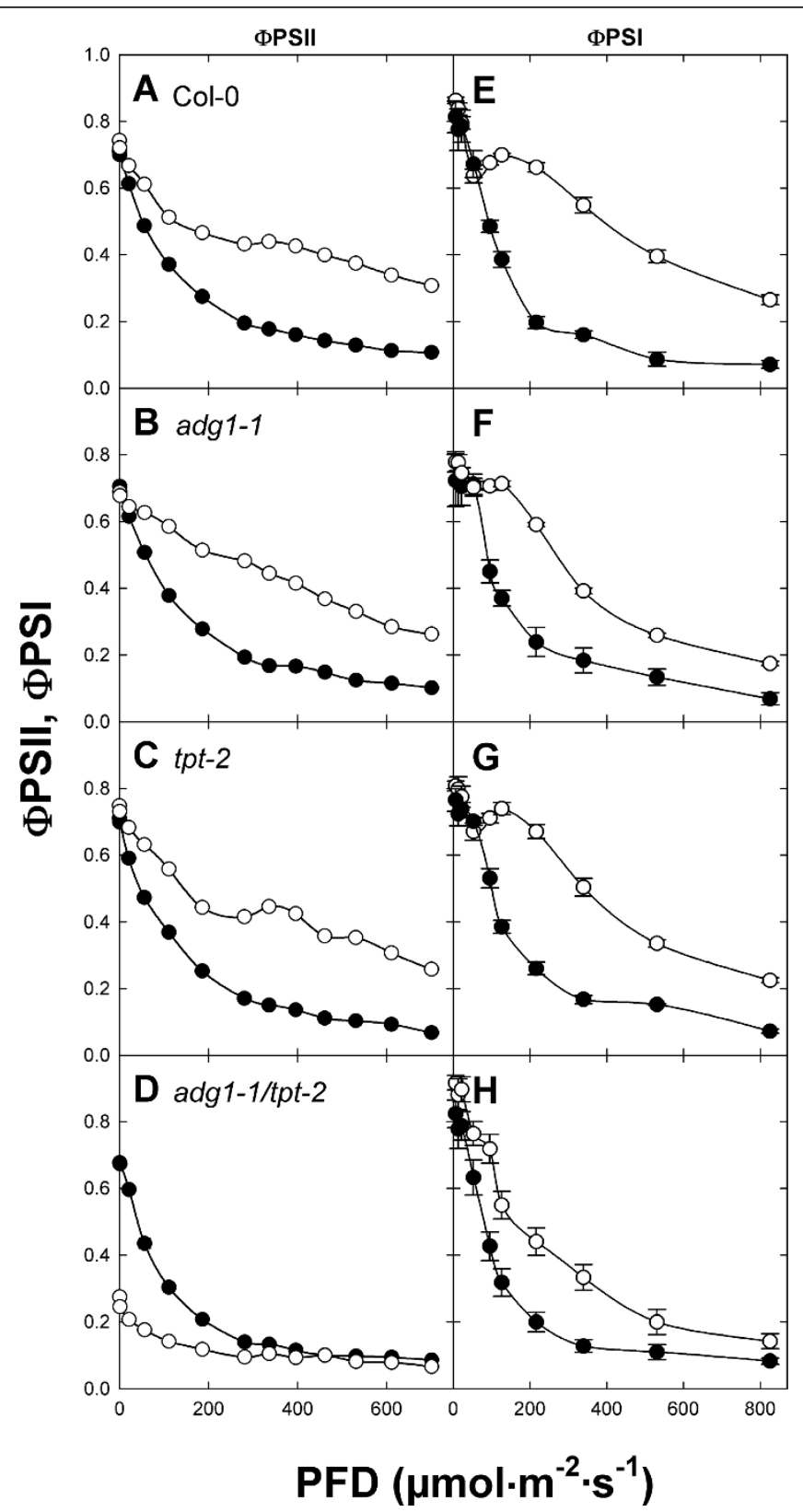

Figure 6 Light dependencies of $\Phi$ PSII and $\Phi P S I$ of HL- and LL-grown wild-type and mutant plants. The response of the quantum efficiencies of PSII (ФPSII, A-D) or PSI (ФPSI, E-H) towards increasing light intensities was determined with a DUAL PAM fluorometer for Col-0 wild type (A, E), the adg1-1 (B, F) or tpt-2 (C, G) single mutants as well as the adg1-1/tpt-2 double mutant (D, H) grown in HL (O) or LL $(\bullet)$. The data represent the mean \pm SE of 12 independent measurements. Note that for some data the error bars are smaller than the symbol size

diminished fluorescence yield in AL. However, it is not clear which mechanism is responsible for the decrease in $\mathrm{F}_{\mathrm{o}}$ after the application of FR light to dark-adapted adg1-1/tpt-2 leaves.

We therefore investigated the mechanistic basis for FR dependent quenching of $F_{o}$ in dark-adapted double mutant plants by the application of inhibitors such as nigericin (a protonophor dissipating the transthylakoid proton gradient), dithiothreitol (DTT; a potent inhibitor of the xanthophyll cycle) and tentoxin (a specific inhibitor of thylakoid ATPase). Compared to the control (Figure 8A), the application of nigericin (Figure $8 \mathrm{~B}$ ) and more so of DTT (Figure 8C) to dark-adapted leaves of adg1-1/tpt-2 resulted in an increase in $\mathrm{F}_{\mathrm{o}}$ and concomitantly a further decrease in the $F_{v} / F_{m}$ ratio, whereas in the wild type these parameters remained unaffected by inhibitor treatments. Most strikingly, upon illumination with FR the Chl- $a$ fluorescence yield was enhanced rather than diminished in 


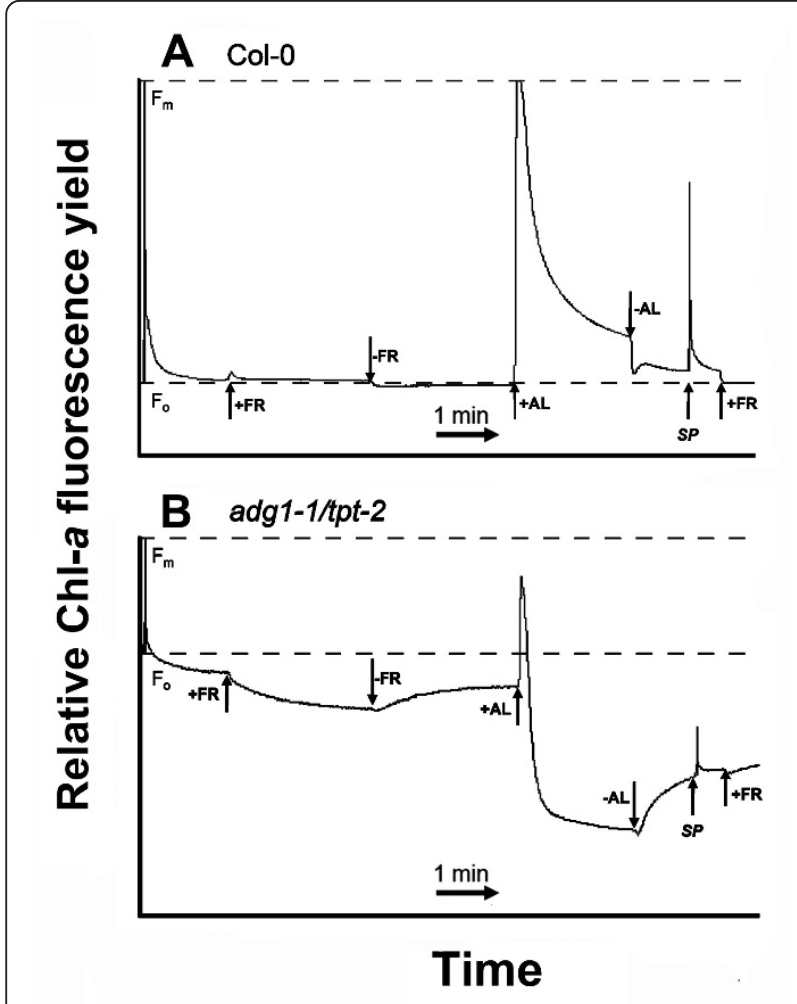

Figure 7 Time course of $\mathrm{Chl}-a$ fluorescence yields of wild-type or double mutant plants in response to far red (FR) or actinic light (AL). The response of Chl-a fluorescence yields of Col-0 wildtype (A) or adg1-1/tpt-2 double mutant plants (B) towards FR or AL (at a PFD of $4000 \mu \mathrm{mol} \cdot \mathrm{m}^{-2} \cdot \mathrm{s}^{-1}$ ) was determined in time course experiments with a PAM 2100 fluorometer. The plants were darkadapted for 30 min prior to the experiment. During the course of the experiment a saturation light pulse (SP) was applied after $5 \mathrm{~s}$ in order to determine $F_{0}$ and $F_{m}$. Where indicated by arrows, FR or AL were either switched on (+) or off (-). SP indicates the application of saturation light pulses

adg1-1/tpt-2 and $\mathrm{F}_{\mathrm{m}}$ was identical to the $\mathrm{F}_{\mathrm{m}}$ of darkadapted leaves both in wild-type and adg1-1/tpt-2 plants. It is likely that the decline in $\mathrm{F}_{\mathrm{m}}$ during FR illumination in the control plants (Figure 8A) is due to the establishment of a proton gradient across the thylakoid membrane. Nonphotochemical energy quenching $\left(\mathrm{q}_{\mathrm{E}}\right)$ of free LHCs induced by this proton gradient is abolished both by nigericin and DTT (Figure 8B and 8C). In contrast, the application of tentoxin resulted in an increase in the $\mathrm{F}_{\mathrm{v}} / \mathrm{F}_{\mathrm{m}}$ ratio of dark-adapted adg1-1/tpt-2 leaves and a more pronounced quenching of $\mathrm{F}_{\mathrm{o}}$ upon illumination with FR light (Figure 8D), suggesting that by inhibition of the thylakoid ATPase a steeper $\mathrm{pH}$ gradient across the thylakoid membrane was established, which enhanced $\mathrm{q}_{\mathrm{E}}$. Figure $8 \mathrm{E}$ shows the time-dependent response of $F_{m}$ ' relative to $F_{m}$ upon illumination with actinic light $\left(\mathrm{PFD}=600 \mu \mathrm{mol} \cdot \mathrm{m}^{-}\right.$ $\left.{ }^{2} \cdot \mathrm{s}^{-1}\right)$. The application of nigericin inhibited $\mathrm{q}_{\mathrm{E}}$ similarly in adg1-1/tpt-2 and the wild type, whereas the application of
DTT had strong inhibitory effects on $\mathrm{q}_{\mathrm{E}}$ in adg1-1/tpt-2 and an intermediate effect in the wild type. The presence of tentoxin had no significant impact on $\mathrm{q}_{\mathrm{E}}$ neither in adg1-1/tpt-2 nor in the wild type (Figure 8E).

Finally, as for adg1-1/tpt-1, deprivation of $\mathrm{O}_{2}$ in the dark-adapted state resulted in a further increase in $F_{o}$ by approximately $10 \%$ in the adg1-1/tpt-2 allele, but not in wild-type plants. This surplus of $F_{o}$ could rapidly be quenched by FR illumination, indicating a re-oxidation of $\mathrm{Q}_{\mathrm{a}}$ and the plastoquinone pool via PSI in the absence of $\mathrm{O}_{2}$ (Additional File 4).

The content of soluble sugars, but not the energy state in leaves is severely compromised in adg1-1/tpt-2 during the light period

A restriction on TP export in the light or the deficiency in starch biosynthesis leads either to an accumulation of starch or soluble sugars $[13,21]$. Hence, a block in both the day- and night-path of photoassimilate export from the chloroplast should result in a severe perturbation of carbohydrate metabolism in leaves of the adg1-1/tpt-2 double mutant. Figure 9 shows variations in contents of starch and soluble carbohydrates (sucrose [Suc], glucose [Glc], and fructose [Fru]) during the course of a day in HL-grown adg1-1/tpt-2 compared to wild-type and single mutant plants. Apart from the deficiency in producing significant amounts of transitory starch (Figure 9A and 9B), adg1-1/tpt-2 is incapable of producing large quantities of soluble carbohydrates during the day (Figure 9D,F and $9 \mathrm{H}$ ). Therefore the double mutant represents a carbohydrate-starved plant. In contrast, the adg1-1 single mutant accumulates soluble sugars, most prominently Glc (Figure 9D). Moreover, small amounts of starch were still detectable both in adg1-1 and the double mutant (Figure 9B). Similar to tpt-1 [13], the tpt-2 allele exhibited increased levels of starch compared to the wild type during the light period (Figure 9A). Furthermore, tpt-2 transiently accumulated Suc within the first 4-5 h in the light, followed by a decline in Suc contents until the end of the light period (Figure 9C). The adg1-1 single mutant showed a similar transient accumulation of soluble sugars during the course of the day (Figure 9D,F and 9H), reflecting most likely restrictions in feedback regulation mechanism of enzymes involved in sucrose biosynthesis in HL. When the plants were grown in LL, the levels of free sugars between the lines remained largely unaffected (Table 2A).

Despite large fluctuations in carbohydrate contents between the plants and a severe growth retardation of HLgrown adg1-1/tpt-2, contents of the adenylates ATP, ADP and AMP remained largely unchanged between the different plant lines grown either under LL- or HL-conditions (Table 2B and 2C). Compared to LL-conditions, the average ATP, ADP, and AMP contents were increased 5-, 6-, 

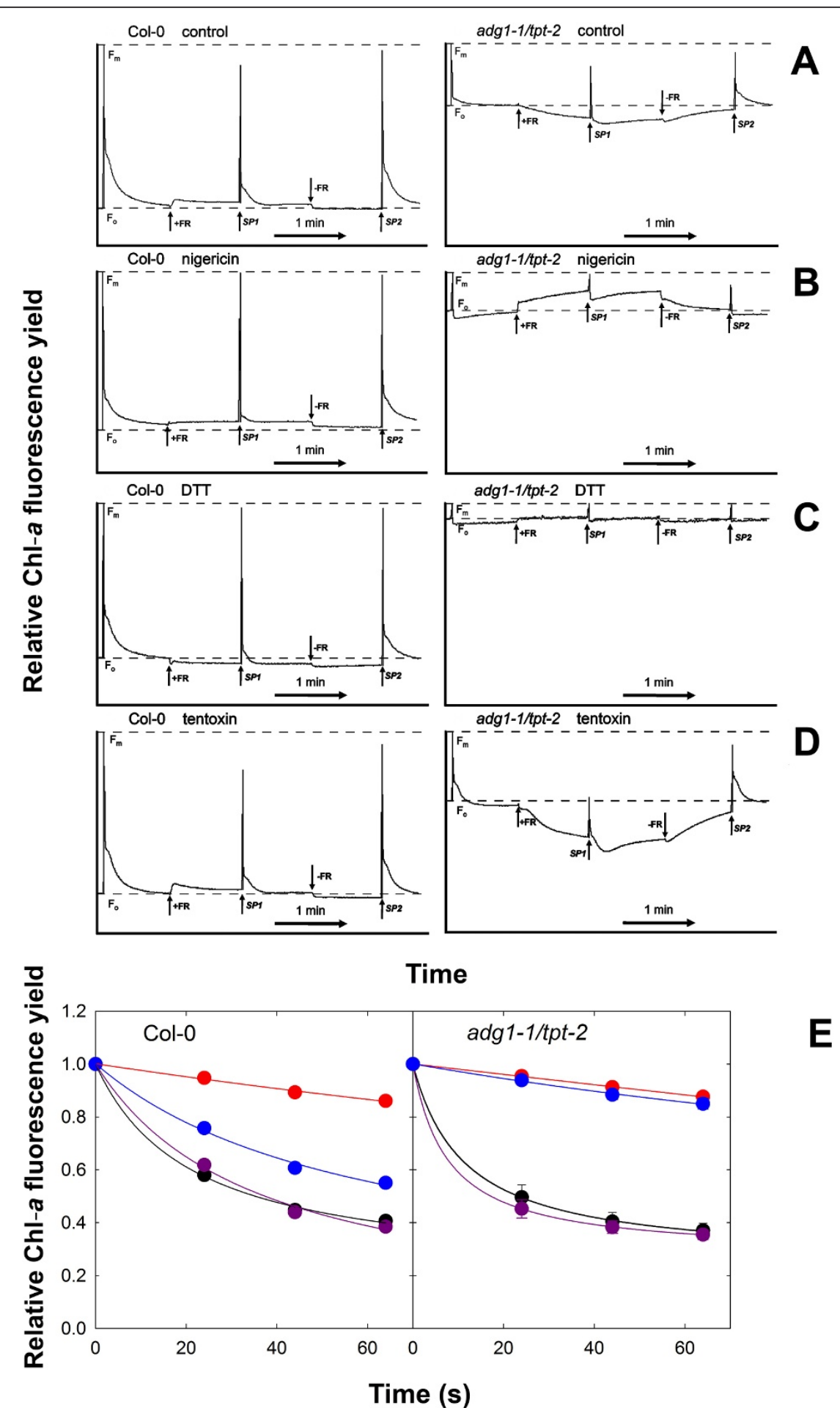

$\mathbf{E}$

Figure 8 Effects of nigericin, DTT and tentoxin on the response of Chl-a fluorescence to illumination with far red light in darkadapted wild-type and double mutant plants. The time course experiments were conducted with a PAM 2100 fluorometer and show the response of Chl-a fluorescence yields of Col-0 wild-type or adg1-1/tpt-2 double mutant plants toward far red (FR) illumination in the absence (control, A) or presence of nigericin (B), DTT (C) or tentoxin (D). Prior to the measurements detached leaves were incubated either in $0.05 \%$ ethanol (control) or inhibitor solutions for $1 \mathrm{~h}$ in the dark. During the course of the experiment a saturation light pulse (SP) was applied after $5 \mathrm{~s}$ in order to determine $F_{\circ}$ and $F_{m}$. Where indicated by arrows, FR was either switched on (+) or off (-). SP indicates the application of saturation light pulses. (E) Impact of nigericin (red circles), DTT (blue circles) or tentoxin (purple circles) on the decay of maximum Chl-a fluorescence yield $\left(\mathrm{F}_{\mathrm{m}}{ }^{\prime}\right)$ in leaves of Col-0 and $a d g 1-1 / \mathrm{tpt}-2$ plants during illumination with AL at a PDF of $600 \mathrm{\mu mol} \cdot \mathrm{m}^{-2} \cdot \mathrm{s}^{-1}$ compared to the control (black circles). The data for adg1-1/tpt-2 represent the mean \pm SE of $n=3$ independent experiment 


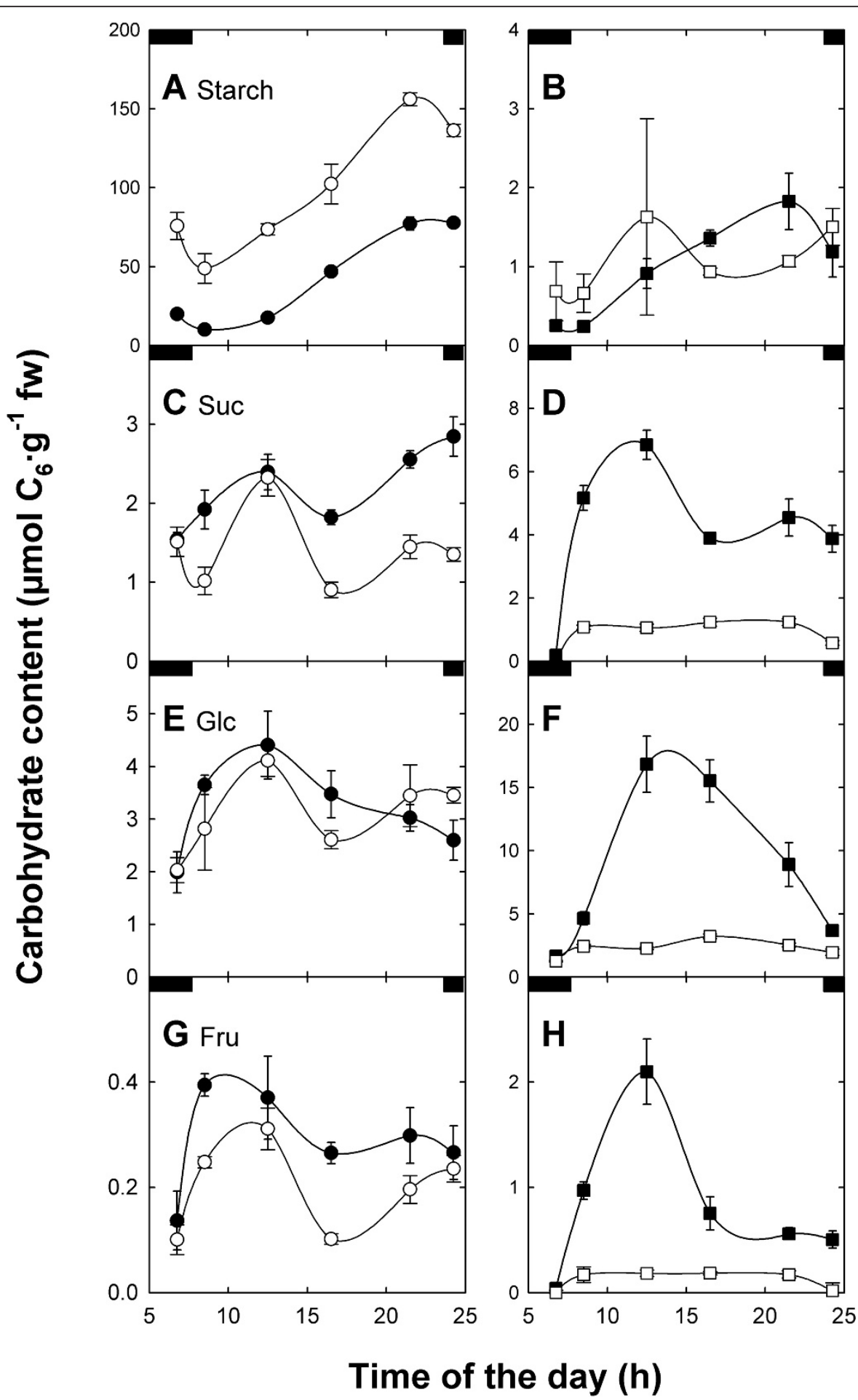

Figure 9 Diurnal changes in carbohydrate contents in leaves of wild-type and mutant plants. Contents of starch (A, B), the soluble sugars sucrose $(C, D)$, glucose $(E, F)$ and fructose $(G, H)$ in leaves of Col-0 wild-type $(\bullet)$, the tpt-2 (0) and adg 1-1 single mutants (- $)$ as well as the adg 1$1 /$ tpt-2 double mutant ( $\square$ ). The data represent the mean \pm SE of three independent experiments. Note that the $y$-axes have been adapted to maximum carbohydrate contents in the individual lines 
Table 2 Carbohydrate contents of LL-grown plants (A) as well as contents of adenylates in Col-0, adg1-1, tpt-2 and adg1-1/tpt-2 grown in LL (B) or HL (C)

\begin{tabular}{|c|c|c|c|c|}
\hline Plant lines & Starch & Sucrose & Glucose & Fructose \\
\hline \multicolumn{5}{|c|}{$\left(\mu \mathrm{mol} C 6 \cdot \mathrm{g}^{-1} \mathrm{fw}\right)$} \\
\hline \multicolumn{5}{|c|}{ A Plants grown in LL } \\
\hline Col-0 & $4.69 \pm 0.59^{\mathbf{b}, \mathbf{d}}$ & $1.71 \pm 0.18$ & $1.69 \pm 0.48^{\mathbf{b}}, \mathbf{d}$ & $0.53 \pm 0.13$ \\
\hline adg1-1 & $0.24 \pm 0.06^{a}$ & $1.47 \pm 0.33$ & $2.46 \pm 0.14^{a}, c$ & $1.05 \pm 0.07$ \\
\hline tpt-2 & $3.89 \pm 0.47^{\mathbf{b}, \mathbf{d}}$ & $1.10 \pm 0.14^{d}$ & $1.04 \pm 0.07^{\mathbf{b}, \mathbf{d}}$ & $0.32 \pm 0.13$ \\
\hline adg1-1/tpt-2 & $0.69 \pm 0.54^{a},{ }^{c}$ & $1.36 \pm 0.38$ & $1.86 \pm 0.16^{a},{ }^{c}$ & $0.68 \pm 0.19$ \\
\hline Plant lines & ATP & ADP & AMP & $\mathrm{EC}$ \\
\hline \multicolumn{5}{|c|}{$\left(\mathrm{nmol} \cdot \mathrm{g}^{-1} \mathrm{fW}\right)$} \\
\hline \multicolumn{5}{|c|}{ B Plants grown in $\mathrm{LL}$} \\
\hline Col-0 & $34.0 \pm 1.5$ & $14.8 \pm 1.1$ & $1.3 \pm 0.1$ & 0.83 \\
\hline adg1-1 & $48.4 \pm 13.5$ & $21.7 \pm 4.9$ & $1.9 \pm 0.6$ & 0.82 \\
\hline tpt-2 & $30.1 \pm 4.5$ & $12.4 \pm 2.0$ & $1.1 \pm 0.2$ & 0.83 \\
\hline adg1-1/tpt-2 & $36.3 \pm 0.4$ & $16.9 \pm 0.9$ & $1.2 \pm 0.1$ & 0.82 \\
\hline Plant lines & ATP & ADP & AMP & $\mathrm{EC}$ \\
\hline \multicolumn{5}{|c|}{$\left(\mathrm{nmol} \cdot \mathrm{g}^{-1} \mathrm{fw}\right)$} \\
\hline \multicolumn{5}{|c|}{ C Plants grown in $\mathrm{HL}$} \\
\hline Col-0 & $159.5 \pm 10.5$ & $90.8 \pm 5.4$ & $19.9 \pm 1.8^{\mathbf{d}}$ & 0.76 \\
\hline adg1-1 & $191.9 \pm 25.4$ & $90.8 \pm 8.6$ & $14.9 \pm 2.1$ & 0.80 \\
\hline tpt-2 & $208.4 \pm 7.9$ & $106.3 \pm 6.3$ & $17.4 \pm 3.1^{d}$ & 0.79 \\
\hline adg1-1/tpt-2 & $154.5 \pm 11.1$ & $88.0 \pm 4.7$ & $9.0 \pm 1.2^{\text {a }}, c$ & 0.79 \\
\hline
\end{tabular}

Leaf samples were taken after $8 \mathrm{~h}$ in the light. Metabolism in the leaf samples was quenched immediately in $\mathrm{N}_{2}$ during illumination. The data represent the mean \pm SE of $n=3(A)$ or $n=6(B$ and C) measurements. Significant differences in the data pairs were identified by the ANOVA test and further analyzed by the Tukey-Kramer test. The letters as superscripts denote the lines to which a significant difference $(P<0.05)$ was found with $a=C o l-0, b=a d g 1-1, c=t p t-2$ and $d=$ adg1-1/tpt-2. The energy charge (EC) was calculated from the relationship $E C=([A T P]+0.5 \cdot[A D P]) /([A T P]+[A D P]+[A M P])$.

and 12 -fold in all plant lines grown in HL. However, taking the energy charge (EC) as a measure of adenylate pool homeostasis into account, there were only little differences between LL- and HL-grown plants. In all plant lines, the EC of between 0.82 and 0.83 at LL was only slightly higher compared to the EC of between 0.76 and 0.80 at HL. Hence, energy deficiency seems not to be the cause for the observed growth and photosynthesis phenotypes of HL-grown adg1-1/tpt-2.

\section{Sugar feeding rescued the growth and HCF phenotypes of adg1-1/tpt-2 plants independently from the induction of GPT2}

Carbohydrate starvation in the adg1-1/tpt-2 is correlated with the development of the growth and HCF phenotype under HL conditions. Therefore the impact of external fed sugars on the acclimation response was investigated in HL-grown wild-type and double mutant plants. Both, the growth and HCF phenotypes of adg1-1/tpt-2 could be rescued when the plants were grown on MS medium supplemented with $50 \mathrm{mM}$ Suc (Figure 10C and 10D) or $50 \mathrm{mM}$ Glc (not shown) compared to the unfed controls (Figure 10A and 10B). The rescue of the HCF phenotype of adg1-1/tpt-2 grown on $50 \mathrm{mM}$ sucrose was reflected in significant increases in the $\mathrm{F}_{\mathrm{v}} / \mathrm{F}_{\mathrm{m}}$ ratio, $\Phi$ PSII, Chl contents and the $\mathrm{Chl} a / b$ ratios (Table 3 ). The above parameters almost recovered to wild-type level. The recovery of Suc-grown double mutant plants was accompanied by a substantial increase in D2 protein (PsbD) abundance and moderately enhanced phosphorylation states of Lhcb2, and PsbA/PsbD (Additional file 5), indicating that carbohydrate starvation observed in adg1-1/ tpt-2 might be a main reason for a compromised HL acclimation. In contrast, feeding of Suc in prolonged darkness (i.e. $48 \mathrm{~h}$ ) failed to rescue the HCF phenotype of adg1-1/tpt-2 (not shown).

We have recently demonstrated that the induction of GPT2 at a transcriptional and functional level is correlated with the accumulation of sugars in leaves of mutants defective in starch biosynthesis [21]. Such an additional capacity to transport phosphorylated intermediates across the envelope membrane might compensate for the loss of TPT activity. Indeed, upon Suc feeding a 10-fold increase in GPT2 transcript abundance (determined by qRT-PCR) in adg1-1/tpt-2 compared to only 1.4-fold in the wild type was detected. Hence, recovery of the double mutant's phenotype in the presence of Suc could be entirely based on the induction of GPT2. To test this assumption, we 


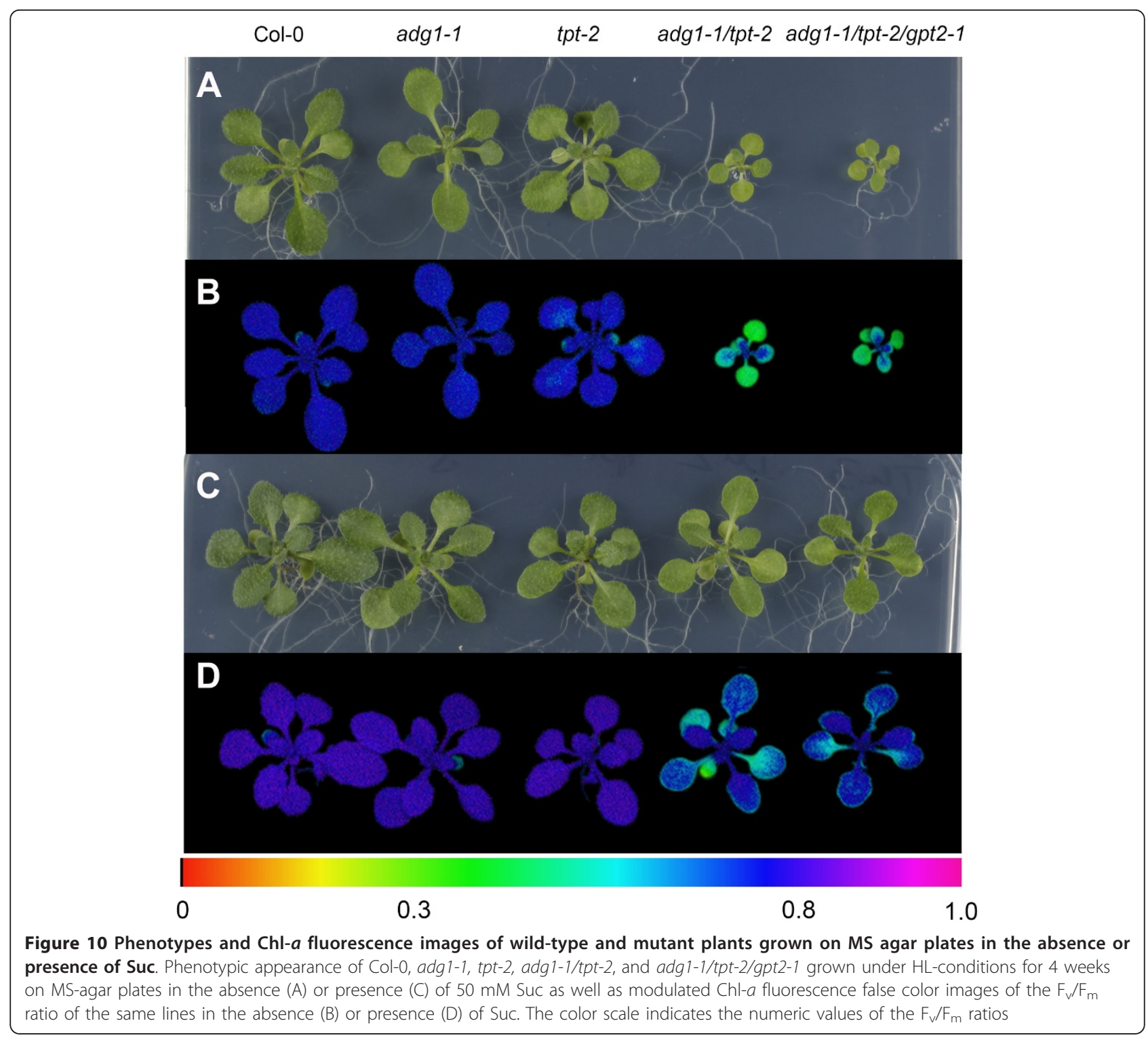

Table 3 Photosynthetic performance as well as pigment contents of Col-0 and adg1-1/tpt-2 plants grown on agar plates containing $1 / 2$ strength MS medium (A) or 1/2 MS supplemented with $50 \mathrm{mM}$ Suc (B) under HL-conditions

\begin{tabular}{|c|c|c|c|c|c|}
\hline Plant line & $\mathrm{F}_{\mathrm{v}} / \mathrm{F}_{\mathrm{m}}$ ratio & ФPSII & Chl content $\left(\mathrm{mg} \cdot \mathrm{g}^{-1} \mathrm{fw}\right)$ & Chl $a / b$ ratio & Carotenoid content $\left(\mu \mathrm{g} \cdot \mathrm{g}^{-1} \mathrm{fw}\right)$ \\
\hline \multicolumn{6}{|c|}{ A Plants grown on $1 / 2$ MS } \\
\hline Col-0 & $0.76 \pm 0.01$ & $0.34 \pm 0.03$ & $1.43 \pm 0.10$ & $3.02 \pm 0.14$ & $51.3 \pm 4.0$ \\
\hline adg1-1/tpt-2 & $0.35 \pm 0.10^{\mathbf{b}}$ & $0.06 \pm 0.05^{a}$ & $0.85 \pm 0.14^{\mathbf{b}}$ & $2.22 \pm 0.08^{a}$ & $34.4 \pm 6.0^{c}$ \\
\hline \multicolumn{6}{|c|}{ B Plants grown on $1 / 2 \mathrm{MS}+50 \mathrm{mM}$ Suc } \\
\hline Col-0 & $0.78 \pm 0.01$ & $0.35 \pm 0.02$ & $1.64 \pm 0.21$ & $2.87 \pm 0.06$ & $49.8 \pm 7.7$ \\
\hline adg1-1/tpt-2 & $0.63 \pm 0.06^{c}$ & $0.21 \pm 0.04^{c, c}$ & $1.55 \pm 0.21^{c}$ & $2.66 \pm 0.03^{a}$ & $59.3 \pm 9.0$ \\
\hline
\end{tabular}

Photosynthesis parameters were determined with the Imaging-PAM fluorometer using the integrated light source. $F_{v} / F_{m}$ ratios were determined after the leaves were dark-adapted for at least $15 \mathrm{~min}$. The OPSIl values refer to steady state photosynthesis, which was attained 7-10 min after onset of illumination at a PFD of $300 \mu \mathrm{mol} \cdot \mathrm{m}^{-2} \cdot \mathrm{s}^{-1}$. For pigment and carbohydrate measurements leaves were harvested in the middle of the light period. The data represent the mean $\pm \mathrm{SE}$ of $\mathrm{n}=$ 4 measurements. Significant differences between the data were analyzed according to the Welch test. The letters as superscripts denote ${ }^{a}, a_{P}<0.01,{ }^{b}, b_{P}<0.02$, c, $c_{P}<0.05$. Plain letters = comparison of adg1-1/tpt-2 with Col-0 at the respective growth conditions, italic letters = comparison of adg1-1/tpt-2 plants grown on $50 \mathrm{mM}$ Suc with those grown on $1 / 2 \mathrm{MS}$ as a control. There were no significant differences found between Col-0 grown either on $1 / 2 \mathrm{MS}$ or $50 \mathrm{mM}$ Suc. 
generated a homozygous adg1-1/tpt-2/gpt2-1 triple mutant, which was phenotypically indistinguishable from the adg1-1/tpt-2 double mutant (Figure 10A and 10B). Strikingly, the triple mutant could also be rescued by Suc feeding (Figure 10C and 10D). This observation indicates that GPT2 does obviously not play a key role in the acclimation response to HL of adg1-1/tpt-2 upon Suc feeding.

\section{A general block in the day- and night path of carbon export from the chloroplast leads to HCF and growth phenotypes similar to adg1-1/tpt-2}

The characteristic growth and HCF phenotypes are not restricted to adg1-1/tpt-2, but are also evident in double mutants impaired in the TPT in combination with other steps of starch biosynthesis such as phosphoglucose isomerase (pgi1-1) or phosphoglucomutase (pgm1), i.e. the pgi1-1/tpt-2 and tpt-2/pgm1 double mutants (Additional File 6A). Like $a d g 1-1 / t p t-2$, these double mutants lack any pronounced phenotype when grown in LL (Additional File 6A). Strikingly, the high-starch mutant mex1$2 / t p t-2$ impaired in both the maltose transporter and the TPT also exhibits a phenotype similar to adg1-1/tpt-2 (Additional File 6B). However, this phenotypic similarity is not based on a high starch level per se, as the sex1-3/ tpt-2 double mutant (Additional File 6C), impaired in the GWD protein and the TPT, does not show a pronounced HCF phenotype. A comparison of the Chl- $a$ fluorescence characteristics between the lines revealed that, apart from sex 1-3/tpt-2, all other double mutants showed an increased $F_{o}$, and a similar response towards AL illumination, i.e. a fluorescence quenching below $\mathrm{F}_{\mathrm{o}}$ followed by a slow recovery in the dark (not shown). However, the quenching of $\mathrm{F}_{\mathrm{o}}$ by FR illumination was absent both in sex 1-3/tpt-2 and mex1-2/tpt-2.

The relative contribution of individual fluorescence classes to the total fluorescence yield, i.e. $\mathrm{F}_{\mathrm{o}}$ emitted from LHCs coupled to or uncoupled from their photosystems as well as the portion of $F_{o}$ quenching by FR illumination and the maximum variable fluorescence $\left(F_{m}\right)$ is summarized in Figure 11 and is based on Chl- $a$ fluorescence time course experiments similar to those shown in Figure 7. In adg1-1/tpt-2, adg1-1/tpt-2/gpt2-1, tpt-2/pgm1 and mex1$2 / t p t-2, F_{m}$ was severely diminished compared to the wild type or the sex 1-3/tpt-2 double mutant, whereas the fluorescence of free antennae was enhanced indicating that a severe restriction in leaf carbohydrate metabolism promotes a massive loss of PSII integrity under HL-conditions. Following application of a very high intensity of AL $\left(\mathrm{PFD}=4000 \mu \mathrm{mol} \cdot \mathrm{m}^{-2} \cdot \mathrm{s}^{-1}\right)$ the $\mathrm{F}_{\mathrm{o}}$ ' (i.e. the fluorescence emission from antennae associated with their phostosystems) was, apart from mex $1-2 / t p t-2$, very similar in all lines compared to the wild type. In the latter double mutant $\mathrm{F}_{\mathrm{o}}$ ' was 1.75 -fold higher than in the wild-type. Moreover, in all starch-free or low-starch double mutants

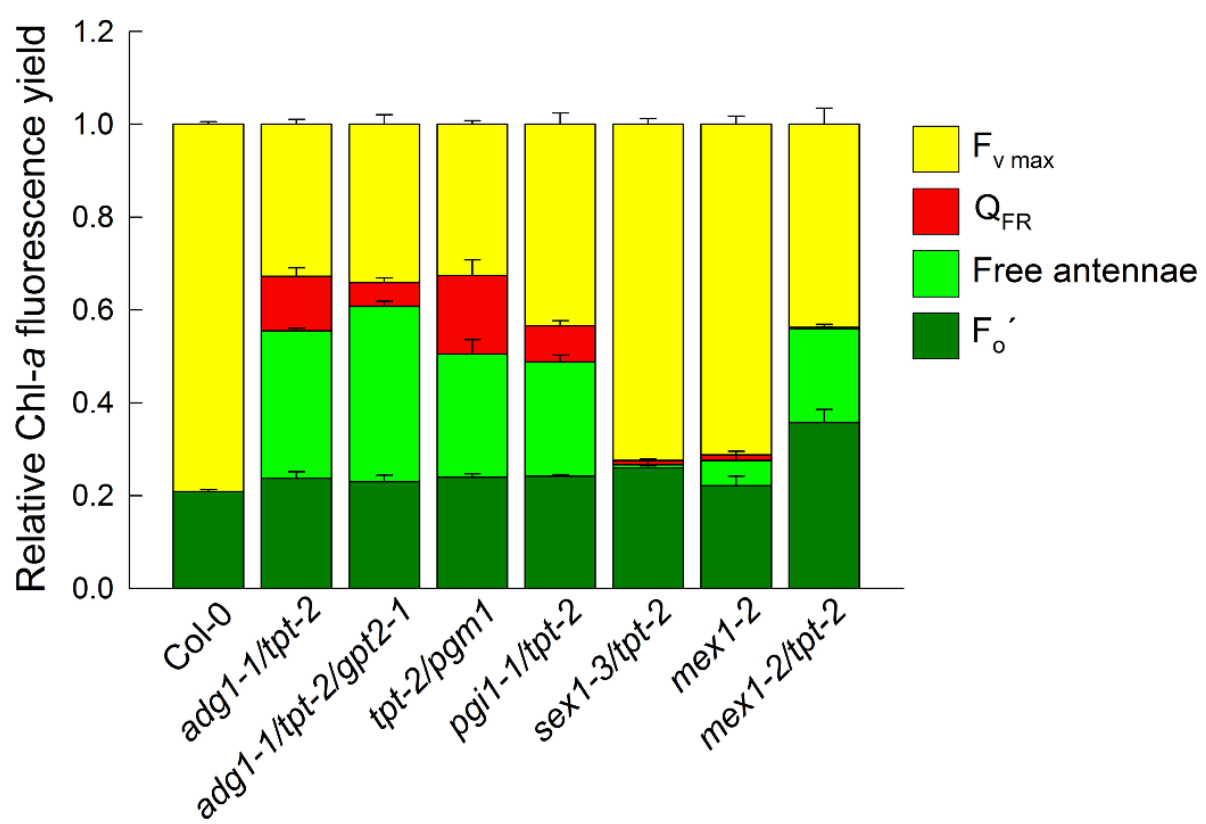

Figure 11 Summarizing analysis of $\mathrm{Chl}-\boldsymbol{a}$ fluorescence yields of dark-adapted wild-type and mutant plants. The analysis is based on similar time course experiments as shown in Figure 7. The individual bars indicate fluorescence emitted from LCHs associated with PSII (dark green bars; $F_{0}$, i.e. after application of AL at a PFD of $4000 \mu \mathrm{mol} \cdot \mathrm{m}^{-2} \cdot \mathrm{s}^{-1}$ ), whereas (light green bars) and (red bars) indicate the relative fluorescence yield emitted from free antennae, which either can (light green bars) or cannot (red bars) be partially quenched by FR illumination $\left(Q_{F R}\right)$. The yellow bars represent the maximum $F_{v}$ emitted from PSII reaction centers. The data were normalized for $F_{m}(=1)$ and represent the mean \pm SE of $n=3$ measurements 
a substantial part of the HCF could be quenched by FR light, most likely by thermal dissipation of excitation energy. In contrast, FR light had no effect on the HCF of mex1-2/tpt-2.

\section{Discussion}

In this report we have studied the acclimation response of plants with a strongly perturbed leaf carbohydrate metabolism. The major outcome of our investigations can be summarized as follows: (i) double mutants impaired in the day- and night path of photoassimilate export are still viable, (ii) the growth and HCF phenotypes develop only when the mutant plants were grown in HL and were virtually absent in LL-grown plants. (iii) Growth in HL resulted in severe changes in the composition of thylakoid proteins involved in photosynthetic electron transport characterized most prominently by a decrease in the plastome-encoded core components of both photosystems leading to increased contents of uncoupled, highly fluorescent nuclear encoded PSII and PSI antennae. Loss of both PSII and PSI reaction centers led to diminished $\mathrm{Chl}$ contents and a drop of the Chl $a$ / $b$ ratio in adg1-1/tpt-2. (iv) Feeding of soluble sugars such as Suc or Glc rescued the growth and HCF phenotypes of the adg1-1/tpt-2 double mutant and the adg11/tpt-2/gpt2-1 triple mutant indicating that either sugar signaling is involved in the altered HL acclimation in carbohydrate-starved plants or alternative NADPH and ATP consuming metabolic pathways are enhanced. Moreover, GPT2 is not a key player in the sugar-triggered rescue of the mutant's phenotype.

\section{Residual TPT activity or the induction of GPT2 is not responsible for survival of double mutants impaired in the day- and night path of photoassimilate export}

A block in starch biosynthesis and the day path of photoassimilate export results in growth retardation and a HCF phenotype of the plants (i.e. adg1-1/tpt-2, tpt-2/ pgm1, pgi1-1/tpt-2). It has previously been proposed that residual TPT activity (which can be as high as 16\% compared to the wild type) in the background of adg1-1/tpt-1 [21] might permit survival of this double mutant allele $[13,20]$. With the aid of the newly established adg1-1/tpt2 double mutant, which completely lacks $T P T$-specific transcripts and TPT transport activity, we could clearly demonstrate that the TPT is not required for survival. It is likely that the induction of other phosphate translocators can compensate for the absence of the TPT. For instance, one of the two A. thaliana GPT genes (i.e. GPT2) is induced in leaves of mutants defective in starch biosynthesis (such as adg1-1, pgm1 and pgi1-2) as well as in the adg1-1/tpt-1 double mutant [21]. It has been proposed that GPT2 is an essential component of acclimation to HL in A. thaliana [28]. In wild-type plants both
GPT genes are either not (GPT2) or only weakly (GPT1) expressed in photoautotrophic tissues [29]. As GPT proteins are capable of transporting TPs besides of Glc6P [30], the presence of a functional GPT in chloroplasts would relief the constraints of photoassimilate export by the TPT. With the aid of the adg1-1/tpt-2/gpt2-1 triple mutant we could demonstrate that GPT2 is not involved in the survival of the double mutant. However, it is conceivable that a small portion of TPs can be exported via the xylulose 5-P (Xu5P)/phosphate translocator (XPT, At5g17630; [31]), which is, according to the electronic fluorescent protein browser, expressed in autotrophic and heterotrophic tissues of $A$. thaliana (http://www.bar. utoronto.ca/efp/cgi-bin/efpWeb.cgi; [32]). Besides its specific transport capacity for Xu5P, the XPT accepts TP, but not 3-PGA as a substrate with a $\mathrm{K}_{\mathrm{m}}$ value in a physiological range (i.e. $0.4 \mathrm{mM}$; [31]). There is, however, no indication that the XPT gene is upregulated in the double mutant background (unpublished data). Future experiments have to clarify whether adg1-1/tpt-2/gpt2-1/xpt quadruple mutants are still viable.

\section{The HCF phenotype of adg1-1/tpt-2 is caused by antennae uncoupled from diminished PSII and PSI core proteins}

Mutants impaired in the day and night path of carbon export from the chloroplasts (i.e. adg1-1/tpt-2, tpt-2/ pgm1, pgi1-1/tpt-2) develop a HCF phenotype when grown in HL. The high $\mathrm{F}_{\mathrm{o}}$ is accompanied by a significant quenching of the fluorescence yield below $F_{o}$ during illumination with AL. These pattern of fluorescence changes is reminiscent of PSII mutants [33], for instance defective in PSII stability [34] or its assembly, such as lpa1 [35], lpa2 [36], or most recently in the thylakoid protein PAM68 [37]. Based on the fluorescence emission spectra at $77 \mathrm{~K}$ and immunoblot analyses, we could demonstrate that the major part of HCF in HL-grown adg1-1/tpt-2 plants derives from changes in the composition or contents of thylakoid proteins The most prominently decreased proteins were the plastome-encoded core proteins of both photosystems (D1, D2, CP43, CP47, P700 apoprotein A2), with the exception of the Cyt $b_{559}$ subunit PsbE and the $\beta$-subunit of thylakoid ATPase (AtpB), whereas the contents of their LHCs or other nuclearencoded proteins such as PsbO or PetC (Rieske) remained rather unchanged or were even increased (FNR). Free antennae are highly fluorescent $[38,39]$ and give rise to the observed HCF phenotype.

Due to the inevitable formation of reactive oxygen species, PSII is constantly damaged, particularly under HL-conditions [40]. D1 and D2 exhibit a high protein turnover, as they are subject to PSII photoinhibition (reviewed by [41-43]). In adg1-1/tpt-2 double mutant plants exposed to HL-conditions, the decomposition 
rate of PSII core proteins presumably exceeds the rate of their de novo synthesis. Moreover, PSI abundance in adg1-1/tpt-2 declined in line with PSII. The basis of the reduced PSI content is still unknown. Normally, PSI content remains unaffected in response to PSII photoinhibition $[44,45]$.

Strikingly, the abundance of $\mathrm{Cyt}_{559}$, a plastomeencoded constituent of PSII remained high despite of a severe decline in D1 or D2. Normally, the content of Cyt $b_{559}$ is strictly correlated with the other PSII core proteins in chloroplasts, and free $C_{y t} b_{559}$ does not accumulate. In case of PSII photoinhibition, Cyt $b_{559}$ is degraded in parallel with the rest of the PSII reaction center [46]. However, free Cyt $b_{559}$ has for instance been observed in etioplasts prior to PSII assembly during de-etiolation $[47,48]$ or in a screen for HCF mutants, particularly those affected in PSII [33]. It has recently been proposed that $\mathrm{Cyt} \mathrm{b}_{559}$ acts as a plastoquinole oxidase [49]. It remains to be determined if free Cyt $b_{559}$ also has a photoprotective function. Strikingly, a deficiency in $C$ t $b_{559}$ rather than an increased Cyt $b_{559}$ abundance leads to a similar HCF phenotype in a tobacco mutant, which has been interpreted as an overreduction of the plastoquinone pool $[49,50]$. Likewise the HCF phenotype of tobacco plants kept and illuminated for a prolonged time in $\mathrm{CO}_{2}$-free air [51] or of Beta vulgaris plants suffering from iron deficiency [52] has been attributed to a reduced PSII acceptor site.

We have recently proposed that the HCF phenotype observed in the adg1-1/tpt-1 double mutant might also be connected to an increased reduction state of $\mathrm{Q}_{\mathrm{A}}$ and the plastoquinone pool (PQ) in the dark [20]. This assumption appeared reasonable considering that deprivation of $\mathrm{O}_{2}$ or the inhibition of chloroplast alternative oxidase (PTOX), involved in chlororespiration, by octyl gallate $(\mathrm{OG})$ further increased the $\mathrm{F}_{\mathrm{o}}$ in the double mutant, but not in the wild type. This surplus of Chl- $a$ fluorescence yield could readily be quenched by the application of FR light, indicating that indeed electrons residing at the PSII acceptor site were removed efficiently via PSI. A similar $10 \%$ rise in $\mathrm{HCF}$ as a response to $\mathrm{O}_{2}$ deprivation and a rapid drop of the additional fluorescence yield by FR illumination has also been observed for the adg1-1/tpt-2 double mutant allele (see Additional File 4). If this surplus of HCF was based on chlororespiration [53], NDH1 would probably not take part in electron transfer as the NDH1-PSI supercomplex is missing in adg1-1/tpt-2 (Figure 4D, Additional File 2). However, the major portion of the HCF in both the adg1-1/tpt-1 and adg1-1/tpt-2 alleles remained unaffected by FR illumination (compare Figure 7B, and [20]) and is emitted from LHCs decoupled from their photosystems.

\section{Thermal dissipation of excitation energy leads to quenching of HCF below $F_{0}$}

Non-photochemical quenching (NPQ) protects the photosystems (in particular PSII) from overexcitation by increased thermal dissipation in the PSII antennae $[54,55]$, which requires the acidification of the thylakoid lumen, the protonation of LHCs [56]) as well as PsbS [57] and an operational xanthophyll cycle, e.g. [58]. These mechanisms were proposed to change the conformation of PSII antenna proteins and PsbS [59-61], which leads to a decline in the lifetime of excited Chl molecules within the antennae $([62,63])$ and results in a higher probability of thermal dissipation rather than excitation transfer to the reaction centers.

Here we provide evidence that the fluorescence of free LHCs can be quenched by thermal dissipation both in actinic light and even in FR light. The kinetics of slow fluorescence decay (in a minutes scale) during FR illumination and its slow recovery in the dark suggest conformational changes of the LHC proteins. Time constants in the range of $45 \mathrm{~s}$, as has been observed in adg1-1/tpt-2 (compare Figure 7B), would be typical for such conformational changes [64]. Moreover, the application of the protonophore nigericin and the xanthophylls cycle inhibitor DTT dramatically diminished NPQ in the double mutant background. Strikingly, HCF quenching upon application of FR light was completely abolished in the presence of nigericin and DTT and showed a trend of an increase in the presence of tentoxin, a specific inhibitor of thylakoid ATPase (Compare Figure 8). The application of FR light to dark-adapted wild-type leaves had only little effect on Chl- $a$ fluorescence. From these observations, we conclude that FR illumination is capable of generating a proton gradient, potentially due to cyclic electron transport (CET) around PSI, which leads to some NPQ of free antennae.

Interestingly, with the exception of the mex $1-2 / t p t-2$ double mutant, the $F_{o}$ ' value upon illumination with high PFDs of AL was almost identical in all the mutant lines tested with a comparable HCF phenotype. Hence, this remaining portion of fluorescence yield might reflect the emission from LHCs functionally coupled with their photosystems. In contrast, the mild HCF phenotype in the mex 1-2/tpt-2 double mutant might be based on mechanical lesions of chloroplasts as has recently been shown for the mex 1 single and $d p e 1 / \operatorname{mex} 1$ [6] or tpt-2/ mex1 double mutants [7].

\section{Is the diminished phosphorylation state of thylakoid proteins responsible for grana hyperstacking in the adg1-1/tpt double mutants?}

Plants can adapt to fluctuating light by state transitions [65], which dominate at low light intensities and involve 
the reversible phosphorylation/dephosphorylation of PSII antennae by a specific kinase (STN7; $[66,67])$ and phosphatase (TAP38, $[68,69])$. This leads to decoupling of the phosphorylated PSII antennae from the core of PSII and the lateral movement to and association with PSI. By this mechanism the distribution of excitation energy between the photosystems can be adapted to the requirements of the plants [70]. Furthermore the STN8 kinase is responsible for the phosphorylation of PSII core proteins such as D1 [66,71] and hence involved in the PSII repair cycle $[43,72,73]$ after photoinhibition. Here we could show that the phosphorylation states of Lhcb2 as well as of PsbC, CaS, and PsbA/PsbD, were severely diminished in HL-grown adg1-1/tpt-2 plants compared to the wild type. Whilst the decline in the phosphorylation states of PsbC and PsbA/PsbD correlates well with a decrease in their total contents, the abundance of Lhcb2 remained unaffected in adg1-1/tpt2 and would hence result in a high portion of non-phosphorylated PSII antennae. The decrease in phosphorylated PSII core protein content combined with a diminished Lhcb2 phosphorylation would be consistent with an increased grana stacking in adg1-1/tpt-2. A modified grana stacking has also been observed in $\operatorname{stn} 8$ and $\operatorname{stn} 7$ mutants [74,75]. Moreover, like adg1-1/tpt-1 and adg1-1/tpt-2 alleles, plants grown under LL-conditions [76-78] or light qualities that favor the excitation of PSI [79] exhibit an increased grana stacking. It remains to be solved whether a more oxidized PSII acceptor site in the double mutant triggers the degree of grana stacking.

\section{External supply of sugars rescues the growth and HCF phenotypes of adg1-1/tpt-2}

Externally supplied Suc or Glc could rescue the growth phenotype of adg1-1/tpt-2. This is not surprising as the double mutant is neither capable of accumulating starch nor large amounts of soluble sugars. Hence feeding of sugars to carbohydrate-starved plants ought to provide sufficient carbon precursors for vegetative growth. However, the rescue of the HCF phenotype of the double mutant cannot be easily explained by a supply of carbon skeletons as an additional energy source for ATP biosynthesis. The energy charge (EC) of LL- and HL-grown mutant and wild-type plants remained largely unchanged in the light, suggesting that a limitation of ATP (e.g. for the PSII repair cycle) is not a major reason for the HCF phenotype or the observed retardation in growth. Strikingly, in the absence of light, feeding of sugars was not effective in alleviating the HCF phenotype of adg1-1/tpt2 (not shown). It is conceivable that the absence or very low levels of soluble sugars blocks signaling pathways required for a proper acclimation response. Hence sugar signaling, i.e. via hexokinase [80] might be directly or indirectly involved in this response, whereas the involvement of GPT2 in the sugar-dependent rescue of the adg1-1/tpt-2 growth and HCF phenotypes can be ruled out as a similar rescue occurred also in the absence of GPT2 in the adg1-1/tpt-2/gpt2-1 triple mutant. Moreover, application of sugars or a changing light environment alters the metabolic signature in the mesophyll, which might itself be recognized as a signal $[81,82]$. Apart from its role as signaling molecule the presence of Glc (or Suc) might enhance metabolic sequences within the chloroplast that act as NADPH and ATP sinks in the light such as biosynthesis of the photosynthetic machinery or de novo fatty acid biosynthesis and thereby relieve photoinhibition.

\section{Conclusions}

Here we demonstrate that carbohydrate limitation compromises acclimation to HL in A. thaliana. It is conceivable that the strong relationship between the chloroplast and nucleus with respect to a co-ordinated expression of photosynthesis genes is modified. This is shown by the severe repression of plastome-encoded subunits of the photosynthetic complexes and the unaltered accumulation of nuclear-encoded components such as LHCs and the subunits of the oxygen evolving complex. The generation and/or transmission of chloroplast signals to the nucleo/ cytosolic system might be strongly altered during HLexposure of adg1-1/tpt-2 compared to the wild type or the single mutants. Therefore, the adg1-1/tpt-2 double mutant may serve as an excellent tool to study principles of retrograde signaling essential for the coordinated expression of nuclear- and plastome-encoded photosynthesis genes in the future. This aspect will be addressed by transcriptomic and metabolomic approaches. Moreover, it ought to be considered that transcription and translation of plastomeencoded genes $([40,83,84]$ as well as the assembly of the photosystems within the thylakoid membrane are partially controlled by nuclear-encoded factors $[85,86]$.

\section{Methods}

\section{Plant material and growth conditions}

Seeds of A. thaliana ecotypes Ws-2, Col-0, and Ler were obtained from the Nottingham Arabidopsis Stock Centre (NASC). In addition, the following mutant lines defective in the genes indicated were used: gpt2-1 (Atlg61800; [29]), adg1-1 (At5g48300; [16]), sex1-3 (At1g10760; [24]), pgm1 (At5g51820; [22[), pgi1-1 (At4g24620; [23]), and mex1-2 (At5g17520; [5]).

Plants were germinated and grown on soil for approximately 4 weeks in a growth cabinet (Percival, CLF Plant Climatics GmBH, Wertingen, Germany, model AR-36 $\mathrm{L} 3 / \mathrm{HIL}$ ) at a light/dark cycle of $16 \mathrm{~h} / 8 \mathrm{~h}$, a day/night temperature of $22^{\circ} \mathrm{C} / 18^{\circ} \mathrm{C}$ and at relative humidity of $40 \%$. Each of the three levels of the growth cabinet was 
equipped with 14 fluorescence tubes (Osram L18W/840), which could be dimmed to the desired PFD. For growth in $\mathrm{HL}$ or LL, the photosynthetic active PFD was adjusted to $300 \mu \mathrm{mol} \cdot \mathrm{m}^{-2} \cdot \mathrm{s}^{-1}$ or $30 \mu \mathrm{mol} \cdot \mathrm{m}^{-2} \cdot \mathrm{s}^{-1}$ at leaf level and controlled once a week by a LI-COR LI 250 light meter. Feeding experiments with exogenous carbohydrates were performed after seeds were germinated and grown on sterile 1/2 Murashige-Skoog (MS) agar or on 1/2 MS agar supplemented with $50 \mathrm{mM}$ Suc or $50 \mathrm{mM}$ Glc for 4 weeks under HL conditions as described above.

\section{Isolation of the tpt-2 mutant allele and generation of double mutants}

The mutant line N573707 (SALK_073707.54.25.x, Col-0 background) was screened via standard PCR on genomic DNA using g_tpt-2 primers in combination with T-DNA border primers (Additional File 7). The resulting PCR product was sequenced and the T-DNA insertion in the TPT gene (At5g46110) 8 bp downstream of the start ATG was verified. The isolated homozygous tpt- 2 mutant plants were smaller in size and were hence backcrossed to Col-0 wild type. Only those plants of the F2 population were further propagated that carried the tpt-2 allele homozygously and lacked any growth retardation compared to Col-0. The tpt-2 mutant line was crossed to adg1-1, pgm1, pgi1-1, sex1-3 and mex1-2. Immature flowers of homozygous single mutants were emasculated and manually cross pollinated. The crosses of tpt-2 $\times$ adg1-1, pgi1-1, sex 1-3, and mex1-2, and were performed using the adg1-1, pgi1-1, and mex1-2 as the female parent. For the cross of $p g m 1 \times t p t-2$ the $t p t-2$ plant was used as the female parent. Triple mutants lacking TPT, ADG and GPT2 were generated by crossing the double mutants adg1-1/tpt-2 to adg1-1/gpt2-1 [21]. The progenies of the crosses were screened either for starch-free or starch excess phenotypes by iodine staining at the end of a light period or at the end of a prolonged dark period and for T-DNA insertions. For primers used see Additional File 7.

\section{Protein isolation and immunoblot analyses}

Samples for protein isolation and subsequent immunoblot analyses were taken directly in the Percival growth chamber under LL- and HL-conditions after $4 \mathrm{~h}$ of illumination. Total proteins of leaves were extracted as described in [87]. Of the protein extract $10 \mu \mathrm{g}$ was separated by discontinuous $12.5 \%$ SDS-polyacrylamide gelelectrophoresis (PAGE) and electroblotted on polyvinylidene fluoride (PVDF) membranes (Bio-Rad). The membranes were incubated overnight in a casein containing blocking solution and probed with primary antibodies against photosynthesis associated proteins supplied by Agrisera (Vännäs, Sweden). Following incubation with the secondary antibody, i.e. horseradish peroxidase conjugate (Sigma,
St. Louis, Missouri), the proteins were detected by chemoluminescence in the presence of SuperSignal West substrate (Thermo Fisher Scientific) by exposure to a film (CL-XPosure, Thermo Scientific). For the detection of phosphorlylated proteins a specific phospho-threonine antibody (Cell signaling, Beverly, Massachusetts) was used as the primary antibody. As casein is highly phosphorylated, it was replaced by BSA in the blocking solution.

\section{Isolation of thylakoid membranes and blue-native-PAGE}

Native isolation of thylakoid membranes was performed according to [88] with $10 \mathrm{mM} \mathrm{NaF}$ added to the extraction media. Membrane proteins were solubilized with $2 \%$ $\mathrm{n}$-dodecyl- $\beta$-D-maltoside in solubilization buffer $(50 \mathrm{mM}$ Bis-Tris [pH 7.0], $750 \mathrm{mM}$ 6-aminocaproicacid; $5 \mathrm{mM}$ EDTA [pH 7.0]; $50 \mathrm{mM} \mathrm{NaCl).} \mathrm{The} \mathrm{samples} \mathrm{were} \mathrm{sepa-}$ rated on commercially available continuous $3-12 \%$ native Bis-Tris acrylamide gels (Invitrogen) as described in [89] and stained with colloidal Coomassie G [90]. For the identification of protein supercomplexes, proteins separated by BN-PAGE in a first dimension were further separated by SDS-PAGE in the second dimension according to [41].

\section{Mass spectroscopic analysis of proteins}

For the further separation and identification of protein supercomplexes on $\mathrm{BN}$-gels, individual protein bands were excised, subjected to a tryptic digestion and the peptides separated by nano-liquid chromatography (Proxeon, Odense, Dänemark), ionized by electrospray ionisation (ESI) and detected and fragmented by tandem MS (HCT Ultra ETD II, Bruker Daltonik, Bremen). The obtained mass and fragment spectra were compared to the NCBInr database (A. thaliana) with the help of Mascot (Matrixscience; [91]) at a significance level of $(P<0.05)$ and a mass tolerance of $\pm 0.3 \mathrm{Da}$.

\section{RNA extraction and qRT-PCR}

RNA was extracted according to [92]. After treatment with DNA-free DNase (Ambion), oligo(dt)-primed cDNA of total RNA was synthesized using the Bioscript reverse transcriptase (Bioline). GPT2 transcript abundance was analyzed by quantitative RT-PCR with the SYBR Green PCR Master Mix (Applied Biosystems) in combination with the 7300 Sequence Detection System (Applied Biosystems). Relative transcript amounts were quantified with the $\mathrm{AAC}_{\mathrm{t}}$ method [93]. As a control ubiquitin C (UBC, At5g25760) was used according to [94]. For gene specific primers see Additional File 7.

\section{Metabolite determination}

Starch and soluble sugars were isolated from frozen leaf material according to [16] and determined with a coupled enzymatic assay [95] in a Spectrafluor Plus plate reader (TECAN, Switzerland) in the absorbance mode. For the 
determination of metabolites with a high turnover such as nucleotides, care was taken that metabolism in the leaf samples was quenched immediately in liquid $\mathrm{N}_{2}$ during illumination with LL or HL. Nucleotides were extracted from around $100 \mathrm{mg}$ of leave material using perchloric acid. After derivatization with chloracetaldehyd the remaining etheno-adensosine nucleotides were separated and quantified by HPLC (Dionex Ultimate LC 3000) as described by [96].

\section{Determination of pigment and protein contents}

Plant material was ground in liquid nitrogen and pigments associated with photosynthesis were extracted in $100 \%$ acetone. Chl content as well as $\mathrm{Chl} a / b$ ratios were assayed in $80 \%$ acetone and calculated as described in [97]. Total carotenoids were determined at a wavelength of $480 \mathrm{~nm}$ and the contents calculated using the equation, $\mathrm{Car}=\mathrm{E}_{480}+0.144 \cdot \mathrm{E}_{663}-0.638 \cdot \mathrm{E}_{645}$. For total protein content measurement, plant material was extracted in $50 \mathrm{mM}$ Hepes- $\mathrm{NaOH}$ ( $\mathrm{pH}$ 7.0) in the presence of $0.1 \%$ Triton-X-100. Protein contents were determined as described in [98].

\section{Determination of TPT transport activity}

TPT transport activity was determined after reconstitution of membrane proteins extracted from A. thaliana leaves into artificial liposomes, according to the method described by [99] using radiolabeled ${ }^{32} \mathrm{P}_{\mathrm{i}}$ as a counter exchange substrate.

\section{In vivo determination of PSII and PSI performance}

Performance of PSII was determined by Chl- $a$ fluorescence measurements with the pulse amplitude modulation fluorometers Imaging-PAM or PAM-2100 (Walz, Effeltrich, Germany). The individual fluorescence parameters determined by the 'saturation-pulse-method' are defined according to [100]. For inhibitor studies excised leaves were incubated for $1 \mathrm{~h}$ in aqueous solutions containing $6.5 \mu \mathrm{M}$ tentoxin, $100 \mu \mathrm{M}$ nigericin [101] or $3 \mathrm{mM}$ DTT [102] in $0.01 \%(\mathrm{v} / \mathrm{v})$ ethanol. As a control leaves were incubated in a $0.01 \%(\mathrm{v} / \mathrm{v})$ ethanol solution.

Performance of PSI was determined from light-induced absorption changes at a wavelength of $830 \mathrm{~nm}$ minus 870 nm [103] with the Dual-PAM (Walz, Effeltrich, Germany). PSI can be completely oxidized by the application of FR light followed by a saturating light pulse (SP), yielding the $P_{m}$ value, which is analogous to $F_{m}$ at PSII. During illumination with actinic light (AL) the reduction state of PSI varies depending on the PFD as well as on acceptor- or donor site limitations. The portion of still oxidizable PSI in the light $\left(\mathrm{P}_{\mathrm{m}}{ }^{\prime}\right)$ can be determined by the application of SPs during AL illumination. The performance of PSI is derived from the three quantum efficiencies ФNA (acceptor site limitation), ФND (donor site limitation), and ФPSI (electron transport). The sum of these three parameters equals one. The quantum efficiencies of electron transport is defined as $\Phi P S I=1-\Phi N D-\Phi N A$.

\section{Thylakoid membrane isolation, quantification of photosynthetic complexes and Chl- $a$ fluorescence emission analysis at $77 \mathrm{~K}$}

Thylakoid membranes were isolated according to [104]. The contents of Cyt $b_{559}$, which can be usually used to quantify PSII, and of the Cyt $b_{6} / f$ complex were determined from difference absorption signals of $C_{y t} b_{559}$, $f$ and $\mathrm{b}_{6}$. Thylakoids equivalent to $50 \mu \mathrm{g} \mathrm{Chl} \cdot \mathrm{ml}^{-1}$ were destacked in a low salt medium, to improve the optical properties of the sample [105]. All cytochromes were oxidized by the addition of $1 \mathrm{mM}$ potassium ferricyanide (+III), and subsequently reduced by addition of $10 \mathrm{mM}$ sodium ascorbate and dithionite, resulting in the reduction of Cyt $\mathrm{f}$ and the high-potential form of $C_{4 t} b_{559}$ (ascorbate-ferricyanide difference spectrum) and of Cyt $b_{6}$ and the low-potential form of Cyt $b_{559}$, respectively. At each redox potential, absorption spectra were measured between 575 and 540 nm wavelength with a V-550 spectrophotometer (Jasco GmbH, Groß-Umstadt, Germany) equipped with a headon photomultiplier. The spectral bandwidth was $1 \mathrm{~nm}$ and the scanning speed was $100 \mathrm{~nm} \cdot \mathrm{min}^{-1}$. Difference absorption spectra were deconvoluted using reference spectra and difference extinction coefficients as in [105]. PSII contents were calculated from the sum of the high-and lowpotential difference absorption signals of Cyt $b_{559}$ [106]. The content of redox-active PSI was determined from light-induced difference absorption changes of $\mathrm{P}_{70} 0$, the PSI reaction center Chl- $a$ dimer. Isolated thylakoids equivalent to $50 \mu \mathrm{g} \mathrm{Chl} \cdot \mathrm{ml}^{-1}$ were solubilized with $0.2 \%$ $(\mathrm{w} / \mathrm{v}) \mathrm{n}$-dodecyl- $\beta$-D-maltoside in the presence of $100 \mu \mathrm{M}$ paraquat as electron acceptor and of $10 \mathrm{mM}$ sodium ascorbate as electron donor. $\mathrm{P}_{700}$ was oxidized by the application of a saturating light pulse $(2000 \mu \mathrm{mol}$ photons $\cdot \mathrm{m}^{-2} \cdot \mathrm{s}^{-1}$ red light, $200 \mathrm{~ms}$ duration). Measurements were done using the Dual-PAM instrument (Walz, Effeltrich, Germany) in its PC-P 700 version [107].

Chl- $a$ fluorescence emission spectra at $77 \mathrm{~K}$ were measured on isolated thylakoids equivalent to $10 \mu \mathrm{g}$ $\mathrm{Chl} \cdot \mathrm{ml}^{-1}$ using a F-6500 fluorometer (Jasco GmbH, Groß-Umstadt, Germany). The samples were excited at $430 \mathrm{~nm}$ wavelength (10 $\mathrm{nm}$ bandwidth). The emission spectra between 655 and $800 \mathrm{~nm}$ were recorded with a bandwidth of $1 \mathrm{~nm}$ and a scanning speed of $200 \mathrm{~nm}$. Ten spectra were averaged, to increase the signal to noise ratio, and corrected for the instrumental response of the photomultiplier.

\section{Transmission electron microscopy}

Pieces of fully developed leaves were quickly fixed in $2 \%$ glutaraldehyde in $50 \mathrm{mM}$ phosphate buffer ( $\mathrm{pH}$ 7.2). 
Water was substituted with acetone and subsequently acetone was substituted with Spurr-media [108]. During this process samples were post-fixed and contrasted with $2 \%$ osmium tetroxide and 1\% uranyl acetate. Polymerized Spurr embedded samples were cut with glass knifes on an ultramicrotome $M T-600$, (RMC, Tuson, USA) and mounted on coated copper grids. Ultrathin sections were post stained with lead citrate and uranyl acetate and viewed in an EM10 electron microscope (Carl Zeis, Jena, Germany).

\section{Statistical evaluation of experimental data}

The data are expressed as mean values \pm standard error (SE) of the indicated number of independent measurements. Significant differences between two data sets were analyzed using the Welch-test, which allows for unequal relative errors between two groups of measurements assuming that a Gauss distribution is applicable [109]. Significant differences between more than two data sets were analyzed using ANOVA implemented in Excel. Data sets which passed the ANOVA as significant different were submitted to the post hoc Tukey-Kramer test, which allows the comparison of unequal sample sizes and finds which mean values are significantly different from one another [110] The Tukey-Kramer test was performed with xlstat implemented in Excel. For data plotting, SigmaPlot8.0 for Windows (SPSS Inc.) was used.

\section{Additional material}

Additional file 1: Distribution of 'grana stack number classes' in LL- and HL- grown wild-type and double mutant plants. The number of stacks of 33 to 105 individual grana was determined on TEM images of two to three chloroplasts per line and grouped into classed between 2 and 17 stacks per granum. The number of each class was expressed as percentage of the total number of grana counted. The distribution of grana stack numbers was calculated from (A) 105, (B) 77, (C) 75, and (D) 33 individual grana of LL- and HL-grown Col-0 (A, B) or adg1-1/tpt-2 (C, D).

Additional file 2: Protein composition of the $>1000 \mathrm{kDa}$ supercomplex. Thylakoid proteins were isolated from wild-type plants by Blue-Native PAGE. The respective protein band was cut out and further analyzed by LC/MS2. The ion score represents $-10 \cdot \log (P)$, where $P$ is the probability that an observed match is a random event. Ion scores $>38$ indicate either identity or extensive homology $(P<0.05)$. Proteins marked by an asterisk indicate putative new subunits of the NDH Supercomplex (compare [27]).

Additional file 3: Light dependency of acceptor- and donor site limitation of PSI determined with HL- and LL-grown wild-type and mutant plants. Quantum efficiencies of acceptor (blue circles, $\Phi$ NA) or donor site (red circles, $\Phi$ ND) limitation of HL- and LL-grown Col-0 wildtype (A, E), adg1-1 (B, F) and tpt-2 (C, G) single mutant as well as the adg 1-1/tpt-2 (D, H) double mutant plants obtained from light saturation curves. The data represent the mean \pm SE of 12 independent measurements.

Additional file 4: Effects of $\mathrm{O}_{2}$ deprivation on Chl-a fluorescence. Chl-a fluorescence traces of HL-grown Col-0 and adg 1-1/tpt-2 double mutant plants flushed either with air, i.e. in the presence of $21 \% \mathrm{O}_{2}$ ( $\mathrm{A}$, B), or with $\mathrm{N}_{2}$, i.e. in the absence of $\mathrm{O}_{2}(C, D)$ in a closed Perspex chamber. Where indicated by arrows, FR illumination was either switched on (+FR) or off (-FR). SP indicates the application of saturated light pulsed at a duration of $0.8 \mathrm{~s}$.

Additional file 5: Immunoblots of thylakoid proteins of sucrose-fed wild-type and adg1-1/tpt-2 plants compared to the unfed controls. Immunoblots of photosynthesis associated proteins after separation of 10 $\mu \mathrm{g}$ total protein isolated from $\mathrm{HL}$-grown Col-0 and adg 1-1/tpt-2 double mutant on SDS-PAGE. Plants were grown either in the absence (MS) or presence of $50 \mathrm{mM}$ Suc. $\mathrm{P}^{*}$-Threonin indicates signals obtained following incubation of the blots with a phospho-threonin antibody. The numbers indicate signals from PsbC (1), CaS (2), PsbA/PsbD (3), and Lhcbll (4).

Additional file 6: Phenotypes and $\mathrm{Chl}-a$ fluorescence images of wildtype and mutant plants. Description of data: Phenotypic appearance as well as modulated $\mathrm{Chl}-\mathrm{a}$ fluorescence false color images of the $\mathrm{F}_{\mathrm{v}} / \mathrm{F}_{\mathrm{m}}$ ratio of the same lines grown in HL or LL for 4 weeks. (A) pgi1-1, pgm1, pgi1-/tpt2, tpt-2/pgm1, (B) mex1-2, mex1-2/tpt-2, (C) sex1-3, and sex1-3/tpt-2. The color scale indicates the numeric values of the $F_{v} / F_{m}$ ratios.

Additional file 7: Oligonucleotide primers. Primers used for the identification of T-DNA mutants by PCR or transcript amounts by RT-PCR or $\mathrm{QRT}$-PCR (RL).

\section{Acknowledgements}

We like to thank the Deutsche Forschungsgemeinschaft for funding this project in the framework of the research unit 804 'Retrograde Signaling of Plants'. Furthermore we like to thank Dr. Brigitte Buchen, University of Bonn, for her additional help with the TEM.

\section{Author details}

'University of Cologne, Botanical Institute, Biocenter Cologne, Zülpicher Str. 47B, D-50674 Cologne, Germany. ${ }^{2}$ Max Planck Institute of Molecular Plant Physiology, Am Mühlenberg 1, D-14476 Potsdam-Golm, Germany. ${ }^{3}$ Universität Bayreuth, Zellbiologie/Elektronenmikroskopie NW I/B1, Universitätsstrasse 30, D-95447 Bayreuth, Germany. ${ }^{4}$ Biozentrum der LudwigMaximilians-Universität München, Department Biologie I - Botanik Großhaderner Str. 2-4, D-82152 Planegg-Martinsried, Germany.

\section{Authors' contributions}

JS contributed to the major part of this work such as PS measurements (PAM 2100, DUAL-PAM, Imaging PAM), immunoblots, Blue-Native gelelectrophoresis, carbohydrate determinations, preparations of samples for TEM as well as the molecular characterization of mutant and double mutant plant. MAS carried out Chl fluorescence measurements with isolated thylakoids at $77 \mathrm{~K}$ as well as spectroscopic determinations of thylakoid electron transport components. SK performed the determination of adenylates. SG was involved in the TEM analyses. AS originally isolated the tpt-2 mutant and established a homozygous line. TK performed the ANOVA analysis and together with $\mathrm{DL}$ helped with the immunoblot analyses of thylakoid proteins. KB crossed a variety of mutant plants and established double and triple mutant lines. UIF and REH conceived the project and participated in the design of the study and its coordination. All authors read and approved the final manuscript.

Received: 2 September 2011 Accepted: 16 January 2012 Published: 16 January 2012

\section{References}

1. Fliege R, Flügge UI, Werdan K, Heldt HW: Specific transport of inorganic phosphate, 3-phosphoglycerate and triosephosphates across the inner membrane of the envelope in spinach chloroplasts. Biochim Biophys Acta 1978, 502:232-247.

2. Weise SE, Weber AP, Sharkey TD: Maltose is the major form of carbon exported from the chloroplast at night. Planta 2004, 218:474-482.

3. Zeeman SC, Kossmann J, Smith AM: Starch: its metabolism, evolution, and biotechnological modification in plants. Annu Rev Plant Biol 2010, 61:209-234.

4. Weber A, Servaites JC, Geiger DR, Kofler H, Hille D, Gröner F, Hebbeker U, Flügge UI: Identification, purification and molecular cloning of a putative plastidic glucose translocator. Plant Cell 2000, 12:787-801. 
5. Niittylä T, Messerli G, Trevisan M, Chen J, Smith AM, Zeeman SC: A previously unknown maltose transporter essential for starch degradation in leaves. Science 2004, 303:87-89.

6. Stettler M, Eicke S, Mettler T, Messerli G, Hörtensteiner S, Zeeman SC: Blocking the metabolism of starch breakdown products in Arabidopsis leaves triggers chloroplast degradation. Mol Plant 2009, 2:1233-1246.

7. Cho MH, Lim H, Shin DH, Jeon JS, Bhoo SH, Park Yl, Hahn TR: Role of the plastidic glucose translocator in the export of starch degradation products from the chloroplasts in Arabidopsis thaliana. New Phytol 2011, 190:101-112.

8. Lu Y, Sharkey TD: The role of amylomaltase in maltose metabolism in the cytosol of photosynthetic cells. Planta 2004, 218:466-473.

9. Lu Y, Sharkey TD: The importance of maltose in transitory starch breakdown. Plant Cell Environ 2006, 29:353-366.

10. Fettke J, Nunes-Nesi A, Alpers J, Szkop M, Fernie AR, Steup M: Alterations in cytosolic glucose phosphate metabolism affect structural features and biochemical properties of starch-related heteroglycans. Plant Physiol 2008, 148:1614-1629.

11. Fettke J, Eckermann N, Kötting O, Ritte G, Steup M: Novel starch-related enzymes and carbohydrates. Cell Mol Biol (Noisy-le-grand) 2007 52(Suppl):883-904

12. Walters RG, Shepard F, Rogers JJM, Rolfe SA, Horton P: Identifications of mutants of Arabidopsis thaliana defective in acclimation of photosynthesis to the light environment. Plant Physiol 2003, 131:472-481.

13. Schneider A, Häusler RE, Kolukisaoglu U, Kunze R, van der Graaff E, Schwacke R, Catoni E, Desimone M, Flügge Ul: An Arabidopsis thaliana knock-out mutant of the chloroplast triose phosphate/phosphate translocator is severely compromised only when starch synthesis, but not starch mobilisation is abolished. Plant J 2002, 32:685-699.

14. Walters RG, Ibrahim DG, Horton P, Kruger NJ: A mutant of Arabidopsis lacking the triose-phosphate/phosphate translocator reveals metabolic regulation of starch breakdown in the light. Plant Physiol 2004, 135:891-906

15. Häusler RE, Schlieben NH, Schulz B, Flügge UI: Compensation of decreased triosephosphate/phosphate translocator activity by accelerated starch turnover and glucose transport in transgenic tobacco. Planta 1998, 204:366-376.

16. Lin TP, Caspar T, Somerville C, Preiss J: Isolation and characterization of a starchless mutant of Arabidopsis thaliana (L.) Heynh lacking ADPglucose pyrophosphorylase activity. Plant Physio/ 1988, 86:1131-1135.

17. Caspar T, Lin TP, Kakefuda G, Benbow L, Preiss J, Somerville C: Mutants of Arabidopsis with altered regulation of starch degradation. Plant Physiol 1991, 95:1181-1188.

18. Ritte G, Lloyd JR, Eckermann N, Rottmann A, Kossmann J, Steup M: The starch-related R1 protein is an alpha -glucan, water dikinase. Proc Natl Acad Sci USA 2002, 99:7166-7171.

19. Hattenbach A, Müller-Röber B, Nast G, Heineke D: Antisense repression of both ADP-glucose pyrophosphorylase and triose phosphate translocator modifies carbohydrate partitioning in potato leaves. Plant Physiol 1997, 115:471-475.

20. Häusler RE, Geimer S, Kunz HH, Schmitz J, Dörmann P, Bell K, Hetfeld S, Guballa A, Flügge Ul: Chlororespiration and grana hyperstacking: how an Arabidopsis double mutant can survive despite defects in starch biosynthesis and daily carbon export from chloroplasts. Plant Physiol 2009, 149:515-533.

21. Kunz HH, Häusler RE, Fettke J, Herbst $K$, Niewiedomski P, Gierth M, Bell M, Steup M, Flügge UI, Schneider A: The role of plastidial glucose6phosphate/phosphate translocators in vegetative tissues of Arabidopsis thaliana mutants impaired in starch biosynthesis. Plant Biology 2010, 12(Suppl. 1):115-128

22. Kofler H, Häusler RE, Schulz B, Groner F, Flügge UI, Weber A: Molecular characterisation of a new mutant allele of the plastid phosphoglucomutase in Arabidopsis, and complementation of the mutant with the wild-type cDNA. Mol Gen Genet 2000, 263:978-986.

23. Yu TS, Lue WL, Wang SM, Chen J: Mutation of Arabidopsis plastid phosphoglucose isomerase affects leaf starch synthesis and floral initiation. Plant Physiol 2000, 123:319-326.

24. Yu TS, Kofler H, Häusler RE, Hille D, Flügge UI, Zeeman SC, Smith AM, Kossmann J, Lloyd J, Ritte G: The Arabidopsis sex 1 mutant is defective in the R1 protein, a general regulator of starch degradation in plants, and not in the chloroplast hexose transporter. Plant Cell 2001, 13:1907-1918.
25. Sommer F, Drepper F, Hippler M: The luminal helix I of PsaB is essential for recognition of plastocyanin or cytochrome c6 and fast electron transfer to photosystem I in Chlamydomonas reinhardtii. J Biol Chem 2002, 277:6573-6581.

26. Vainonen JP, Sakuragi Y, Stael S, Tikkanen M, Allahverdiyeva Y Paakkarinen V, Aro E, Suorsa M, Scheller HV, Vener AV, Aro EM: Light regulation of $\mathrm{CaS}$, a novel phosphoprotein in the thylakoid membrane of Arabidopsis thaliana. FEBS J 2008, 275:1767-1777.

27. Peng L, Fukao Y, Fujiwara M, Takami T, Shikanai T: Efficient operation of $\mathrm{NAD}(\mathrm{P}) \mathrm{H}$ dehydrogenase requires supercomplex formation with photosystem I via minor LHCl in Arabidopsis. Plant Cell 2009, 21:3623-3640.

28. Athanasiou K, Dyson BC, Webster RE, Johnson GN: Dynamic acclimation of photosynthesis increases plant fitness in changing environments. Plant Physiol 2010, 152:366-373.

29. Niewiadomski P, Knappe S, Geimer S, Fischer K, Schulz B, Unte US, Rosso MG, Ache P, Flügge UI, Schneider A: The Arabidopsis plastidic glucose 6-phosphate/phosphate translocator GPT1 is essential for pollen maturation and embryo sac development. Plant Cell 2005, 17:760-775.

30. Kammerer B, Fischer K, Hilpert B, Schubert S, Gutensohn M, Weber A, Flügge Ul: Molecular characterization of a carbon transporter in plastids from heterotrophic tissues: the glucose 6-phosphate/phosphate antiporter. Plant Cell 1998, 10:105-117.

31. Eicks M, Maurino V, Knappe S, Flügge Ul, Fischer K: The plastidic pentose phosphate translocator represents a link between the cytosolic and the plastidic pentose phosphate pathways in plants. Plant Physiol 2002, 128:512-522

32. Winter D, Vinegar B, Nahal H, Ammar R, Wilson GV, Provart NJ: An "Electronic Fluorescent Pictograph" browser for exploring and analyzing large-scale biological data sets. PLOS ONE 2007, 2:e718.

33. Meurer J, Meierhoff K, Westhoff P: Isolation of high-chlorophyllfluorescence mutants of Arabidopsis thaliana and their characterisation by spectroscopy, immunoblotting and northern hybridisation. Planta 1996, 198:385-396

34. Meurer J, Plücken $\mathrm{H}$, Kowallik KV, Westhoff P: A nuclear-encoded protein of prokaryotic origin is essential for the stability of photosystem II in Arabidopsis thaliana. EMBO J 1998, 17:5286-9527.

35. Peng L, Ma J, Chi W, Guo J, Zhu S, Lu Q, Lu C, Zhang L: Low PSII ACCUMULATION1 is involved in efficient assembly of photosystem II in Arabidopsis thaliana. Plant Cell 2006, 18:955-969.

36. Ma J, Peng L, Guo J, Lu Q, Lu C, Zhang L: LPA2 is required for efficient assembly of photosystem II in Arabidopsis thaliana. Plant Cell 2007, 19:1980-1993.

37. Armbruster U, Zühlke J, Rengstl B, Kreller R, Makarenko E, Rühle T, Schünemann D, Jahns P, Weisshaar B, Nickelsen J, Leister D: The Arabidopsis thylakoid protein PAM68 is required for efficient D1 biogenesis and photosystem II assembly. Plant Cell 2010, 22:3439-3460.

38. Yamane $Y$, Kashino $Y$, Koike $H$, Satoh $K$ : Increases in the fluorescence $F_{0}$ level and reversible inhibition of Photosystem II reaction center by hightemperature treatments in higher plants. Photosynth Res 1997, 52:57-64.

39. Barros T, Royant A, Standfuss J, Dreuw A, Kühlbrandt W: Crystal structure of plant light-harvesting complex shows the active, energy-transmitting state. EMBO J 2009, 28:298-306.

40. Rochaix JD: Assembly of photosynthetic complexes. Plant Physiol 2011 155:1493-1500.

41. Aro EM, Suorsa M, Rokka A, Allahverdiyeva Y, Paakkarinen V, Saleem A Battchikova N, Rintamäki E: Dynamics of photosystem II: a proteomic approach to thylakoid protein complexes. J Exp Bot 2005, 56:347-356.

42. Tyystjärvi E: Photoinhibition of photosystem II and photodamage of the oxygen evolving manganese cluster Source. Coord Chem Rev 2008, 252:361-376.

43. Nixon PJ, Michoux F, Yu J, Boehm M, Komenda J: Recent advances in understanding the assembly and repair of photosystem II. Ann Bot 2010, 106:1-16.

44. Powles SB: Photoinhibition of photosynthesis induced by visible light. Annu Rev Plant Physiol 1984, 35:14-44.

45. Sonoike K: Photoinhibition of photosystem I: Its physiological significance in the chilling sensitivity ot plants. Plant Cell Physiol 1996, 37:239-247.

46. Ortega JM, Roncel M, Losada M: Light-induced degradation of cytochrome b559 during photoinhibition of the photosystem II reaction center. FEBS Lett 1999, 458:87-92. 
47. Müller B, Eichacker LA: Assembly of the D1 precursor in monomeric PSII reaction centre precomplexes precedes $\mathrm{Chl}$ a-triggered accumulation of reaction centre II in barley etioplasts. Plant Cell 1999, 11:2365-2377.

48. Kanervo E, Singh M, Suorsa M, Paakkarinen V, Aro E, Battchikova N, Aro EM: Expression of protein complexes and individual proteins upon transition of etioplasts to chloroplasts in pea (Pisum sativum). Plant Cell Physiol 2008, 49:396-410.

49. Bondarava N, Gross CM, Mubarakshina M, Golecki JR, Johnson GN, KriegerLiszkay A: Putative function of cytochrome b559 as a plastoquinol oxidase. Physiol Plant 2010, 138:463-473.

50. Bondarava N, De Pascalis L, Al-Babili S, Goussias C, Golecki JR, Beyer P, Bock R, Krieger-Liszkay A: Evidence that cytochrome $b_{559}$ mediates the oxidation of reduced plastoquinone in the dark. J Biol Chem 2003, 278:13554-13560.

51. Durchan $M$, Vacha $F$, Krieger-Liszkay A: Effects of severe $\mathrm{CO}_{2}$ starvation on the photosynthetic electron transport chain in tobacco plants. Photosynth Res 2001, 68:203-213.

52. Belkhodja R, Morales F, Quilez R, Lopez-Millan AF, Abadia A, Abadia J: Iron deficiency causes changes in chlorophyll fluorescence due to the reduction in the dark of the photosystem II acceptor side. Photosynth Res 1998, 56:265-276.

53. Rumeau D, Peltier G, Cournac L: Chlororespiration and cyclic electron flow around PSI during photosynthesis and plant stress response. Plant Cell Environ 2007, 30:1041-1051.

54. Horton P, Walters R, Ruban AV: Regulation of light harvesting in green plants. Annu Rev Plant Physiol, Plant Mol Biol 1996, 47:655-684

55. Holt NE, Fleming GR, Niyogi KK: Toward an understanding of the mechanism of nonphotochemical quenching in green plants. Biochemistry 2004, 43:8281-8289.

56. Walters RG, Ruban AV, Horton P: Higher plant light-harvesting complexes LHClla and LHCllc are bound by dicyclohexylcarbodiimide during inhibition of energy dissipation. Eur J Biochem 1994, 226:1063-1069.

57. Li XP, Gilmore AM, Caffarri S, Bassi R, Golan T, Kramer D, Niyogi KK: Regulation of photosynthetic light harvesting involves intrathylakoid lumen $\mathrm{pH}$ sensing by the PsbS protein. J Biol Chem 2004, 279:22866-22874.

58. Jahns P, Latowski D, Strzalka K: Mechanism and regulation of the violaxanthin cycle: the role of antenna proteins and membrane lipids. Biochim Biophys Acta 2009, 1787:3-14.

59. Bergantino E, Segalla A, Brunetta A, Teardo E, Rigoni F, Giacometti GM, Szabò I: Light- and pH-dependent structural changes in the PsbS subunit of photosystem II. Proc Natl Acad Sci USA 2003, 100:15265-15270.

60. Ahn TK, Avenson TJ, Ballottari M, Cheng YC, Niyogi KK, Bassi R, Fleming GR: Architecture of a charge-transfer state regulating light harvesting in a plant antenna protein. Science 2008, 320:794-797.

61. Betterle N, Ballottari M, Zorzan S, de Bianchi S, Cazzaniga S, Dall'Osto L, Morosinotto T, Bassi R: Light-induced dissociation of an antenna heterooligomer Is needed for non-photochemical quenching induction. J Biol Chem 2009, 284:15255-15266.

62. Gilmore AM, Hazlett TL, Govindjee : Xanthophyll cycle-dependent quenching of photosystem II chlorophyll a fluorescence: formation of a quenching complex with a short fluorescence lifetime.anthophyll cycledependent quenching of photosystem II chlorophyll a fluorescence: formation of a quenching complex with a short fluorescence lifetime. Proc Natl Acad Sci USA 1995, 92:2273-2277.

63. Gilmore AM: Xanthophyll cycle-dependent nonphotochemical quenching in Photosystem II: mechanistic insights gained from Arabidopsis thaliana L. mutants that lack violaxanthin deepoxidase activity and/or lutein. Photosynth Res 2001, 67:89-101.

64. Wentworth M, Ruban AV, Horton P: Kinetic analysis of nonphotochemical quenching of chlorophyll fluorescence. 2. Isolated light-harvesting complexes. Biochemistry 2001, 40:9902-9908.

65. Kargul J, Barber J: Photosynthetic acclimation: structural reorganisation of light harvesting antenna-role of redox-dependent phosphorylation of major and minor chlorophyll a/b binding proteins. FEBS J 2008, 275:1056-1068.

66. Bonardi V, Pesaresi P, Becker T, Schleiff E, Wagner R, Pfannschmidt T, Jahns P, Leister D: Photosystem II core phosphorylation and photosynthetic acclimation require two different protein kinases. Nature 2005, 437:1179-1182.
67. Bellafiore S, Barneche F, Peltier G, Rochaix JD: State transitions and light adaptation require chloroplast thylakoid protein kinase STN 7. Nature 2005, 433:892-895.

68. Pribil M, Pesaresi P, Hertle A, Barbato R, Leister D: Role of plastid protein phosphatase TAP38 in LHCII dephosphorylation and thylakoid electron flow. PLOS Biol 2010, 8:e1000288.

69. Shapiguzov A, Ingelsson B, Samol I, Andres C, Kessler F, Rochaix JD, Vener AV, Goldschmidt-Clermont M: The PPH1 phosphatase is specifically involved in LHCII dephosphorylation and state transitions in Arabidopsis. Proc Natl Acad Sci USA 2010, 107:4782-4782.

70. Lemeille S, Rochaix JD: State transitions at the crossroad of thylakoid signalling pathways. Photosynth Res 2010, 106:33-46.

71. Pesaresi P, Pribil M, Wunder T, Leister D: Dynamics of reversible protein phosphorylation in thylakoids of flowering plants: the roles of STN7, STN8 and TAP38. Biochim Biophys Acta 2010, 1807:887-896.

72. Tikkanen M, Nurmi M, Kangasjärvi S, Aro EM: Core protein phosphorylation facilitates the repair of photodamaged photosystem II at high light. Biochim Biophys Acta 2008, 1777:1432-1437.

73. Goral TK, Johnson MP, Brain AP, Kirchhoff H, Ruban AV, Mullineaux CW: Visualizing the mobility and distribution of chlorophyll proteins in higher plant thylakoid membranes: effects of photoinhibition and protein phosphorylation. Plant J 2010, 62:948-959.

74. Fristedt R, Willig A, Granath P, Crèvecoeur M, Rochaix JD, Vener AV: Phosphorylation of photosystem II controls functional macroscopic folding of photosynthetic membranes in Arabidopsis. Plant Cell 2009, 21:3950-3964.

75. Fristedt R, Granath P, Vener AV: A protein phosphorylation threshold for functional stacking of plant photosynthetic membranes. PLoS One 2010, 5:e10963.

76. Lichtenthaler HK, Kuhn G, Prenzel U, Buschmann C, Meier D: Adaptation of chloroplast ultrastructure and of chlorophyll protein levels to high-light and low-light conditions. Z Naturforsch 1982, 37c:464-475.

77. Lichtenthaler HK, Burgstahler R, Buschmann C, Meier D, Prenzel U, Schonthal A: Effect of high light and high light stress on composition, function and structure of the photosynthetic apparatus. In Effects of Stress on Photosynthesis. Edited by: Marcelle R, H. Clijsters H, Van Poucke M. The Hague, Martinus Nijhoff/Dr. W. Junk Publishers; 1983:353-370.

78. Allen JF, Forsberg J: Molecular recognition in thylakoid structure and function. Trends Plant Sci 2001, 6:317-326.

79. Wagner R, Dietzel L, Bräutigam K, Fischer W, Pfannschmidt T: The longterm response to fluctuating light quality is an important and distinct light acclimation mechanism that supports survival of Arabidopsis thaliana under low light conditions. Planta 2008, 228:573-587.

80. Rolland F, Baena-Gonzalez E, Sheen J: Sugar sensing and 46ignalling in plants: conserved and novel mechanisms. Annu Rev Plant Biol 2006, 57:675-709.

81. Bräutigam K, Dietzel L, Kleine T, Ströher E, Wormuth D, Dietz KJ, Radke D, Wirtz M, Hell Rd, Dörmann P, Nunes-Nesi A, Schauer N, Fernie AR, Oliver SN, Geigenberger $P$, Leister D, Pfannschmidt T: Dynamic plastid redox signals integrate gene expression and metabolism to induce distinct metabolic states in photosynthetic acclimation in Arabidopsis. Plant Cell 2009, 21:2715-2732.

82. Pfannschmidt T: Plastidial retrograde signalling-a true "plastid factor" or just metabolite signatures? Trends Plant Sci 2010, 15:427-435.

83. Barkan A, Goldschmidt-Clermont M: Participation of nuclear genes in chloroplast gene expression. Biochimie 2000, 82:559-572

84. Eberhard S, Finazzi G, Wollman FA: The dynamics of photosynthesis. Annu Rev Genet 2008, 42:463-515.

85. Bellafiore S, Ferris P, Naver H, Gohre V, Rochaix JD: Loss of Albino3 leads to the specific depletion of the light-harvesting system. Plant Cell 2002, 14:2303-2314.

86. Sundberg E, Slagter JG, Fridborg I, Cleary SP, Robinson C, Coupland G: ALBINO3, an Arabidopsis nuclear gene essential for chloroplast differentiation, encodes a chloroplast protein that shows homology to proteins present in bacterial membranes and yeast mitochondria. Plant Cell 1997, 9:717-730.

87. Laemmli UK: Cleavage of structural proteins during the assembly of the head of bacteriophage T4. Nature 1970, 227:680-685.

88. Bassi R, dal Belin Peruffo A, Barbato R, Ghisi R: Differences in chlorophyllprotein complexes and composition of polypeptides between thylakoids 
from bundle sheaths and mesophyll cells in maize. Eur J Biochem 1985, 146:589-595.

89. Schägger $\mathrm{H}$, von Jagow $\mathrm{G}$ : Blue native electrophoresis for isolation of membrane protein complexes in enzymatically active form. Anal Biochem 1991, 199:223-231

90. Kang D, Gho YS, Suh M, Kang C: Highly sensitive and fast protein detection with coomassie brilliant blue in sodium dodecyl sulfatepolyacrylamide gel electrophoresis. Bull Korean Chem Soc 2002, 23:1511-1512.

91. Perkins DN, Pappin DJ, Creasy DM, Cottrell JS: Probability-based protein identification by searching sequence databases using mass spectrometry data. Electrophoresis 1999, 20:3551-3567.

92. Logemann J, Schell J, Willmitzer L: Improved method for the isolation of RNA from plant tissues. Anal Biochem 1987, 163:16-20.

93. Ramakers C, Ruijter JM, Deprez RHL, Moorman AFM: Assumption-free analysis of quantitative real-time polymerase chain reaction (PCR) data. Neuroscience letters 2003, 339:62-66.

94. Czechowski T, Stitt M, Altmann T, Udvardi MK, Scheible WR: Genome-wide identification and testing of superior reference genes for transcript normalization in Arabidopsis. Plant Physiol 2005, 139:5-17.

95. Stitt M, Lilley RMC, Gerhardt R, Heldt H: Determination of metabolite levels in specific cells and subcellular compartments of plant leaves. Methods Enzymol 1989, 174:518-552

96. Haink $G$, Deussen A: Liquid chromatography method for the analysis of adenosine compounds. J Chromatogr B Analyt Technol Biomed Life SC 2003, 784:189-193.

97. Arnon DI: Copper enzymes in isolated chloroplasts. Polyphenoloxidase in Beta vulgaris. Plant Physiol 1949, 24:1-15.

98. Schaffner W, Weissmann C: A rapid, sensitive, and specific method for the determination of protein in dilute solution. Anal Biochem 1973, 56:502-514.

99. Flügge $U$, Weber $A$ : A rapid method for measuring organellespecific substrate transport in homogenates from plant tissues. Planta 1994, 194:181-185.

100. Schreiber U, Schliwa U, Bilger W: Continuous recording of photochemical and non-photochemical chlorophyll fluorescence quenching with a new type of modulation fluorometer. Photosyn Res 1986, 10:51-62.

101. Quick P, Scheibe R, Stitt M: Use of tentoxin and nigericin to investigate the possible contribution of $\Delta \mathrm{pH}$ to energy dissipation and the control of electron transport in spinach leaves. Biochim Biophys Acta 1988, 974:282-288.

102. Gamon JA, Field CB, Bilger W, Bjiirkman O, Fredeen AL, Pefiuelas J: Remote sensing of the xanthophyll cycle and chlorophyll fluorescence in sunflower leaves and canopies. Oecologia 1990, 85:1-7.

103. Klughammer C, Schreiber U: An improved method, using saturating light pulses, for the determination of photosystem I quantum yield via P700 ${ }^{+}$-absorbance changes at $830 \mathrm{~nm}$. Planta 1994, 192:261-268.

104. Schöttler MA, Kirchhoff H, Weis E: The role of plastocyanin in the adjustment of the photosynthetic electron transport to the carbon metabolism in tobacco. Plant Physiol 2004, 136:4265-4274.

105. Kirchhoff H, Mukherjee U, Galla HJ: Molecular architecture of the thylakoid membrane: lipid diffusion space for plastoquinone. Biochemistry 2002, 41:4872-4882.

106. Lamkemeyer P, Laxa M, Collin V, Li W, Finkemeier I, Schöttler MA Holtkamp V, Tognetti VB, Issakidis-Bourguet E, Kandlbinder A, Weis E, Miginiac-Maslow M, Dietz KJ: Peroxiredoxin Q of Arabidopsis thaliana is attached to the thylakoids and functions in context of photosynthesis. Plant J 2006, 45:968-981.

107. Schöttler MA, Flügel C, Thiele W, Bock R: Knock-out of the plastidencoded PetL subunit results in reduced stability and accelerated leaf age-dependent loss of the cytochrome $b_{6} f$ complex. J Biol Chem 2007, 282:976-985.

108. Spurr AR: A low-viscosity epoxy resin embedding medium for electron microscopy. J Ultrastruct Res 1969, 26:31-43.

109. Welch BL: The generalization of "student's" problem when several different population variances are involved. Biometrika 1947, 34:28-35.

110. Ludbrook J: Multiple comparison procedures updated. Clin Exp Pharmacol Physiol 1998, 25:1032-1037. doi:10.1186/1471-2229-12-8

Cite this article as: Schmitz et al:: Defects in leaf carbohydrate

metabolism compromise acclimation to high light and lead to a high chlorophyll fluorescence phenotype in Arabidopsis thaliana. BMC Plant Biology 2012 12:8.

\section{Submit your next manuscript to BioMed Central and take full advantage of:}

- Convenient online submission

- Thorough peer review

- No space constraints or color figure charges

- Immediate publication on acceptance

- Inclusion in PubMed, CAS, Scopus and Google Scholar

- Research which is freely available for redistribution 